Ronald P. Muraro

EDIS FE526

W. Greg Hartt

W. C. Oswalt

http://edis.ifas.ufl.edu/FE526

\title{
Budgeting Costs and Returns for Central Florida Citrus Production, 2003-04
}
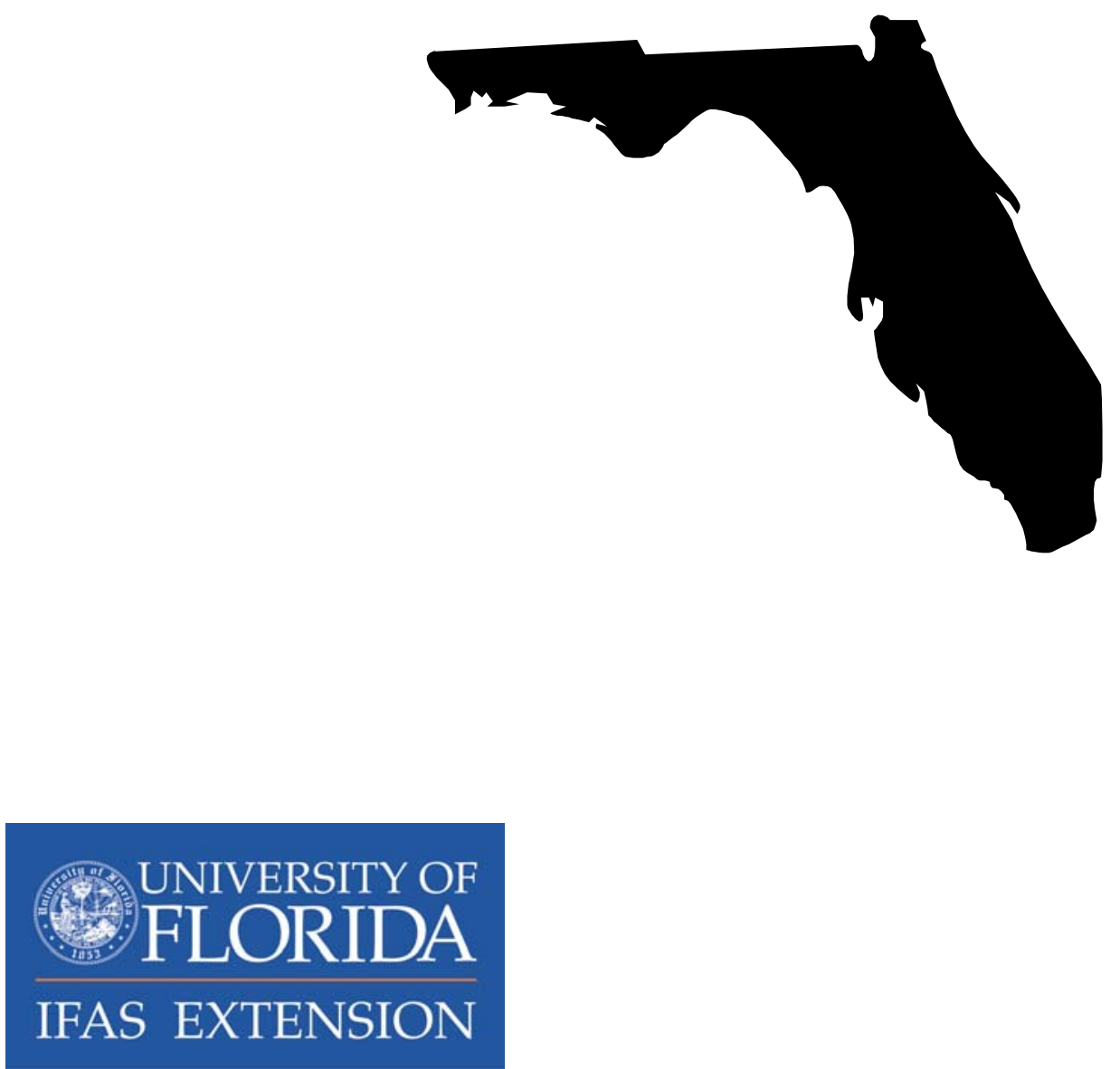

Institute of Food and Agricultural Sciences Food and Resource Economics Department Florida Agricultural Experiment Station Florida Cooperative Extension Service Gainesville, FL 32611 


\begin{abstract}
Estimated costs and returns of growing round oranges in the Central Florida citrus area are presented for the twentieth consecutive year. Due to the freezes of the 1980s, the Central Florida citrus area refers primarily to Polk and Highlands Counties. The format presented may be used by individual growers to budget costs and returns, utilizing individual data on specific groves.
\end{abstract}

Key words: citrus, Central Florida, budgeting, costs and returns

NOTE: The Central Florida production area refers to Polk and Highlands Counties. However, the costs presented in this report are applicable to other counties such as Hardee, Hillsborough, Lake, and Orange.

The budgeted cost information presented herein is the most current available. The budget cost items have been revised to reflect current grove practices being used by growers (e.g., chemical mowing, different spray materials and rates of fertilization, microsprinkler irrigation, more reset trees, etc.). Thus, the 2003-04 budget costs reflect lower fertilizer and pesticide materials costs and increased per acre yield due to higher per acre tree densities.

The budget costs in this report represent a custom-managed operation. Therefore, all equipment costs are based on average custom rate costs along with a 10 percent handling and supervision charge added to the material cost.

Although the estimated annual per acre grove costs listed are representative of a mature citrus grove $(10+$ years old), the grove care costs for a specific grove site may differ depending on tree age, tree density, and grove practices performed (e.g., spot herbicide for grass/brush regrowth under trees could add an additional $\$ 19.23$ per acre; Diaprepes control could add $\$ 73.20$ per acre for each foliar application; extensive tree loss due to blight or tristeza could substantially increase the tree replacement and care costs; spray applications to control citrus leafminer and nematicide applications, such as Temik at $\$ 122.22$ per acre, could increase the total cultural costs per acre above the average costs shown in the comparative budgets; or travel and set-up costs may vary due to size of the citrus grove and distance from grove equipment barn and could add $\$ 25.98$ per acre).

\title{
ACKNOWLEDGMENTS
}

Appreciation is extended to Mrs. Jane Wilson for typing the final draft of this manuscript. Appreciation is also extended to the citrus growers and production managers of the Central Florida citrus production area who provided suggestions for the revision of this manuscript. 


\section{TABLE OF CONTENTS}

$\underline{\text { Page }}$

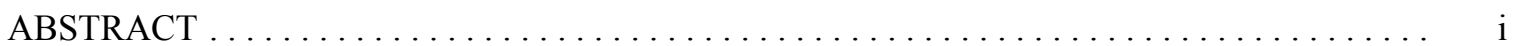

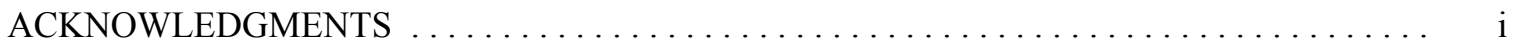

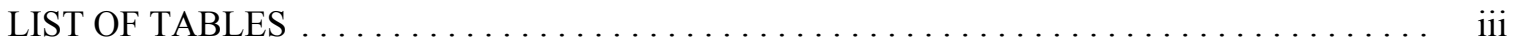

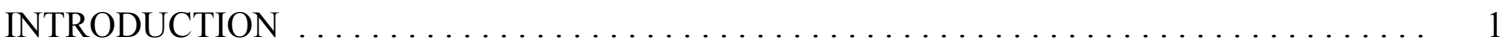

METHOD OF DATA COLLECTION $\ldots \ldots \ldots \ldots \ldots \ldots \ldots \ldots \ldots \ldots \ldots \ldots \ldots \ldots \ldots \ldots \ldots \ldots$

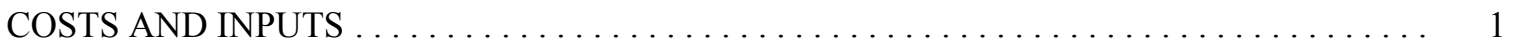

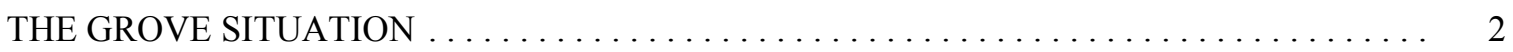

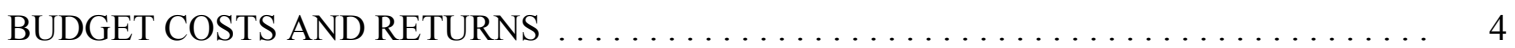

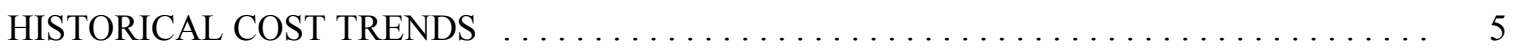

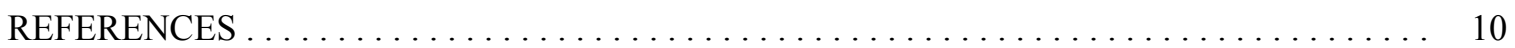

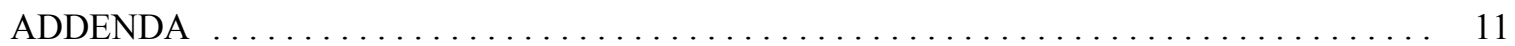

NOTE: The ADDENDA includes items such as Listing of Grove Care Programs for Central Florida Citrus Production for Both Round Oranges and Grapefruit; 2004 custom rate summary report; cost of establishing a citrus grove; etc. Page 11 lists the tables included in the ADDENDA. 


\section{LIST OF TABLES}

Table

$\underline{\text { Page }}$

1 Schedule of production practices and budget items for a Central Florida citrus grove, 2003-04

2 Calculation of normal production per acre, $2003-04 \ldots \ldots \ldots \ldots \ldots \ldots \ldots \ldots$

3 Estimated annual per acre costs and returns for a mature, Valencia orange grove producing for the processed market, Central Florida area, 2003-04 . . . . . . . . . 6

4 Estimated total delivered-in cost for Central Florida (Ridge) Valencia oranges grown for the processed juice market under three cultural cost programs, 2003-04 . . . . . . . . .

5 Estimated annual per acre costs and returns and 5-year average costs and returns for a mature, Valencia orange grove producing citrus for processing in the Central Florida area, 1999-00-2003-04 . .

6 Estimated annual per acre costs and returns and 5-year average costs and returns (adjusted to 2004 dollars) for a mature, Valencia orange grove producing citrus for processing in the Central Florida area, 1999-00-2003-04 


\title{
BUDGETING COSTS AND RETURNS FOR CENTRAL FLORIDA CITRUS PRODUCTION, 2003-04
}

\author{
Ronald P. Muraro, W. Greg Hartt and W. C. Oswalt
}

\section{INTRODUCTION}

Budget analysis provides the basis for many grower decisions. Budget analysis can be used to calculate potential profits from an operation, to determine cash requirements for an operation, or to determine break-even prices. This report presents a budget constructed from current data and serves as a format for growers to analyze costs and returns from their individual records. During the 1980s, several freezes occurred which changed the character of the Central Florida citrus production area. The December 1983 and January 1985 freezes caused extensive tree and acreage losses in north central counties such as Lake and Orange. The December 1989 freeze resulted in severe tree damage and tree loss in north and central Polk County. Thus, Central Florida in this report refers primarily to Polk and Highlands Counties.

\section{METHOD OF DATA COLLECTION}

The data presented here were developed by surveying custom operators, input suppliers, growers, colleagues at the Citrus Research and Education Center in Lake Alfred, and County Extension citrus agents in the Central Florida production region. The survey is conducted annually in February and March.

\section{COSTS AND INPUTS}

Costs for various production inputs are those collected from citrus growers as well as the average of the data obtained from annual custom rate, chemical, and fertilizer surveys. The ADDENDA shows grower's costs (Tables 1-A through 7-A), custom rate costs (Table 8-A), and various chemical and

Ronald P. Muraro, Professor and Extension farm management economist, Department of Food and Resource Economics, Citrus Research and Education Center, Lake Alfred; W. Greg Hartt, Extension citrus agent, Highlands County, Sebring; .and W.C. Oswalt, multi-county Extension citrus agent, Polk and Hillsborough Counties, Bartow; Florida Cooperative Extension Service, Institute of Food and Agricultural Sciences, University of Florida, Gainesville, FL. 
fertilizer costs (Tables 9-A and 10-A). The budget costs represent a custom-managed operation. Therefore, all equipment costs are based on the average custom-rate costs along with a 10 percent handling and supervision charge added to the material cost.

Although brand names are used in many of the tables in the ADDENDA, this does not imply endorsement by the University of Florida. It is merely an attempt to depict typical production practices.

All tables have a column reserved for individual growers to insert data from a particular grove. This will allow a comparison of the grower's costs with those presented.

\section{THE GROVE SITUATION}

Production practices for a Central Florida round orange grove are shown in Table 1 along with times during the year when they are normally performed. There are two benefits to developing such a table for an individual grove. First, it shows what work is needed and when so that operations can be planned well in advance. Second, an annual cash flow analysis can be helpful in financial planning. The individual grower may benefit from developing a plan for a particular grove.

Specific production practices vary from grove to grove, making it difficult to define a "typical" grove. Many combinations of practices and various tree variety combinations produce acceptable yields and returns. Although the example represents a Valencia orange grove, the cost and return data are designed to be applicable to most grove situations. A grower, realtor, or land appraiser can substitute individual grove costs and expected returns into the budget format to develop a budget for a particular grove. A "your cost" column is appropriately provided for this purpose in subsequent tables.

In the following budget, above average management and cultural practices are assumed. Beyond this general assumption, the following specifics are assumed.

1. A $10+$ year-old, low volume-irrigated grove.

2. Variety is Valencia round orange.

3. Tree loss is three percent annually.

4. Trees are pulled and replaced when production falls below 50 percent of expected yield.

5. Production is for processed use.

6. Tree density is 112 trees per acre.

7. Custom-caretaker is providing grove management. 


\begin{tabular}{|c|c|c|c|c|c|c|c|c|c|c|c|c|c|}
\hline & & \multicolumn{12}{|c|}{ Month } \\
\hline & & Jan & Feb & Mar & Apr & May & Jun & Jul & Aug & Sep & Oct & Nov & Dec \\
\hline \multicolumn{2}{|l|}{ Total revenue: } & & & $\begin{array}{c}20 \% \\
\text { deposit } \\
\end{array}$ & & $\begin{array}{c}50 \% \\
\text { Partial } \\
\text { payment }\end{array}$ & & & & & & & $\begin{array}{c}\text { Final } \\
\text { payment }\end{array}$ \\
\hline \multirow[t]{2}{*}{ Less: } & Pick \& haul cost & & & $\mathrm{X}$ & & & & & & & & & \\
\hline & DOC advertisement tax & & & $\mathrm{X}$ & & & & & & & & & \\
\hline \multicolumn{14}{|c|}{ Grove expenses: } \\
\hline \multicolumn{2}{|l|}{ Disc } & & & $\mathrm{X}$ & & & & & & & $\mathrm{X}$ & & \\
\hline \multicolumn{14}{|l|}{ Chop } \\
\hline \multicolumn{2}{|l|}{ Mow } & & & & & $\mathrm{X}$ & & $\mathrm{X}$ & & $\mathrm{X}$ & & & \\
\hline \multicolumn{2}{|c|}{ Labor, general grove work, pull vines } & $\mathrm{X}$ & & & & & & & & $\mathrm{X}$ & & & \\
\hline \multicolumn{2}{|c|}{ Herbicide ( $1 / 2$ grove acre equivalent) } & & & $\mathrm{X}$ & & & $\mathrm{X}$ & & & & & & \\
\hline \multirow[t]{5}{*}{ Spray: } & Post bloom/nutritional & & & & $\mathrm{X}$ & & & & & & & & \\
\hline & Summer oil/greasy spot & & & & & & & $\mathrm{X}$ & & & & & \\
\hline & Fall miticide & & & & & & & & & & $\mathrm{X}$ & & \\
\hline & Supplemental miticide & & & & & & & & & & & & \\
\hline & Dust & & & & & & & & & & & & \\
\hline \multicolumn{2}{|c|}{ Fertilizer } & & \multicolumn{2}{|c|}{$68 \# \mathrm{~N} / \mathrm{A}$} & & \multicolumn{2}{|c|}{$68 \# \mathrm{~N} / \mathrm{A}$} & & & & \multicolumn{2}{|c|}{$68 \# \mathrm{~N} / \mathrm{A}$} & Dolomite \\
\hline \multicolumn{2}{|c|}{ Hedging and topping } & & \multicolumn{3}{|c|}{ Hedge } & & & & & & & & \\
\hline \multicolumn{2}{|c|}{ Brush removal/chop brush } & & \multicolumn{3}{|c|}{ Chop brush } & & & & & & & & \\
\hline \multicolumn{2}{|c|}{ Tree removal } & & & $\mathrm{X}$ & $\mathrm{X}$ & & & & & & & & \\
\hline \multicolumn{2}{|c|}{ Young tree care } & & & $\mathrm{X}$ & $\mathrm{X}$ & & $\mathrm{X}$ & $\mathrm{X}$ & & $\mathrm{X}$ & & & \\
\hline \multicolumn{2}{|c|}{ Microjet irrigation (times/week) } & 1 & 1 & 2 & 3 & 3 & 3 & 2 & 2 & 2 & 2 & 1 & 1 \\
\hline \multicolumn{14}{|c|}{ Miscellaneous } \\
\hline \multicolumn{2}{|c|}{ Grove taxes including water management } & & & & & & & & & & & $\mathrm{X}$ & \\
\hline \multicolumn{2}{|c|}{ Interest expense } & & & & & & & $\mathrm{X}$ & & & & & \\
\hline \multicolumn{2}{|c|}{ Annual principal payment on mortgage } & & & & & & & $\mathrm{X}$ & & & & & \\
\hline
\end{tabular}

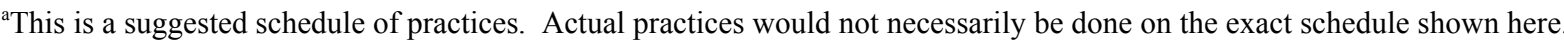


Tree ages will vary due to tree losses and replacement. The budget reflects the following age distribution and yield for Central Florida Valencia oranges:

\begin{tabular}{rlc} 
& \multicolumn{1}{c}{ Situation } & $\begin{array}{c}\text { Yield } \\
\text { Boxes/Tree }\end{array}$ \\
$3 \%$ & pulled and reset trees & 0.0 \\
$3 \%$ & 1-year1old trees & 0.0 \\
$3 \%$ & 2-year-old trees & 0.0 \\
$3 \%$ & 3-year-old trees & 0.7 \\
$3 \%$ & 4-year-old trees & 0.9 \\
$45 \%$ & 5- to 19-year-old trees & 4.2 \\
$3 \%$ & trees producing 50\% of expected yield & 3.1 \\
$37 \%$ & mature producing trees & 6.0
\end{tabular}

Calculation of normal production per acre is shown in Table 2. Note that the proportion-of-treesby-age column only adds up to 91 percent since 9 percent of the trees are non-bearing.

Table 2.--Calculation of normal production per acre, 2003-04

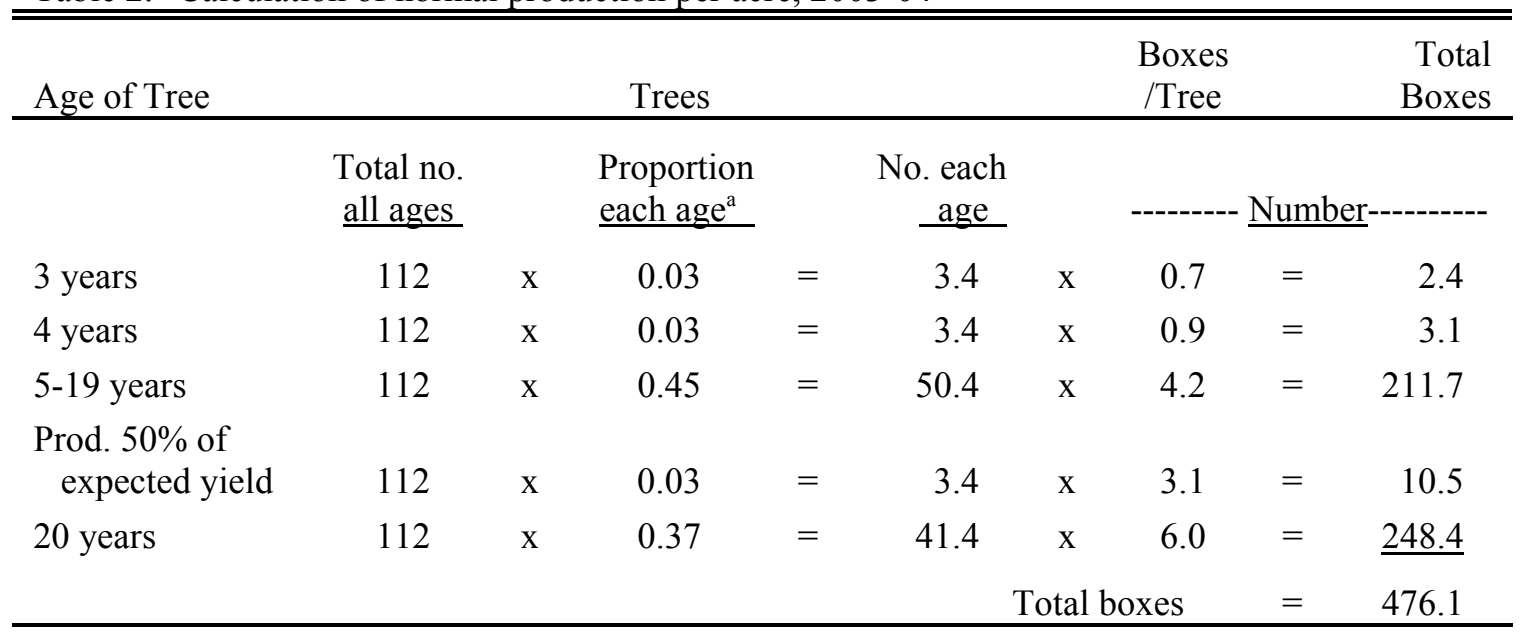

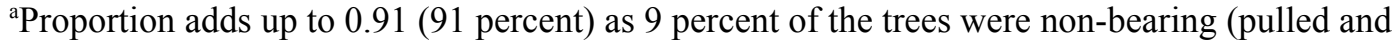
reset, 1- and 2-year-old trees).

\section{BUDGET COSTS AND RETURNS}

Estimated budget costs and returns for a Central Florida grove situation are shown in Table 3. Gross revenue estimates are based on projected yields (Table 3 ) and estimated preliminary on-tree prices for the 2003-04 season. The budgeted costs represent one possible citrus production program and were selected from the costs shown in the ADDENDA tables (grove establishment and reset costs and harvesting and packing charges are shown in Tables 11-A through 14-A and historical on-tree prices for selected Florida citrus varieties are shown in Table 15-A). 
As shown in Table 3, the total revenue for processed-market Valencia oranges is estimated to be $\$ 1,190.00$ per acre. Total specified costs are $\$ 822.18$, comprised of grove care costs of $\$ 774.18$ plus management costs of $\$ 48.00$. A return to land, trees, and ownership of $\$ 367.82$ per acre loss represents net return above variable costs. At 300 and 500 boxes per acre, respectively, the break-even prices required to cover grove care costs for Valencia oranges range from $\$ 2.58$ to $\$ 1.55$ per box on-tree and $\$ 0.74$ to $\$ 0.58$ per pounds solids delivered-in.

Ad valorem taxes, and overhead and administrative costs (e.g., water drainage district taxes, crop insurance, and other grower assessments) can add up to 12 percent of total grove care costs. These costs vary from grove to grove, depending on age, location, or variety of fruit, and should be considered in arriving at net return to land, trees, and ownership (total return minus total costs). Harvest costs (pick, roadside, and hauling costs) also add to the total fruit cost delivered to either a processing plant or fresh fruit packinghouse. Average annual debt payment (principal and interest) may be as high as $\$ 440$ per acre $(\$ 3,750$ average debt per acre at 10 percent interest amortized over 20 years), which would reduce total available cash for grove expansion or other investment.

Estimated "delivered-in" costs for processed oranges are shown in Table 4. "Delivered-in" costs include grove care costs (Table 3) plus harvesting, regulatory, and grower assessment costs. The "delivered-in" cost is presented as a cost per acre, per box and per pound solids. Three possible budget cost scenarios are presented (Table 11-A): Low Cost Processed Cultural Program; Reduced Cost Cultural Program; and Typical/Historical Cultural Program. Scenarios 1 and 2 represent costs of two possible cultural programs directed toward reducing expenditures for fruit grown primarily for the processed market. Scenario 3 represents typical costs of grove practices that have been performed for citrus grown for the fresh/processed fruit market. Modified herbicide and/or spray and fertilizer programs account

for the reduced costs. NOTE: Before modifying a grove management program to reduce costs, an evaluation of the market program (processed or fresh), yield, and specific cultural problems (nutrition, disease, etc.) for the specific grove site should be made.

\section{HISTORICAL COST TRENDS}

Annual budgets of costs and returns for mature, processed Valencia oranges in the Central Florida area have been developed and published the past four years. Estimated cost and return histories for 1999-00 through 2002-03 along with 2003-04 and a five-year average are presented in Table 5. To allow comparisons in current values, these same costs and returns (adjusted to 2004 dollars) are presented in Table 6. 


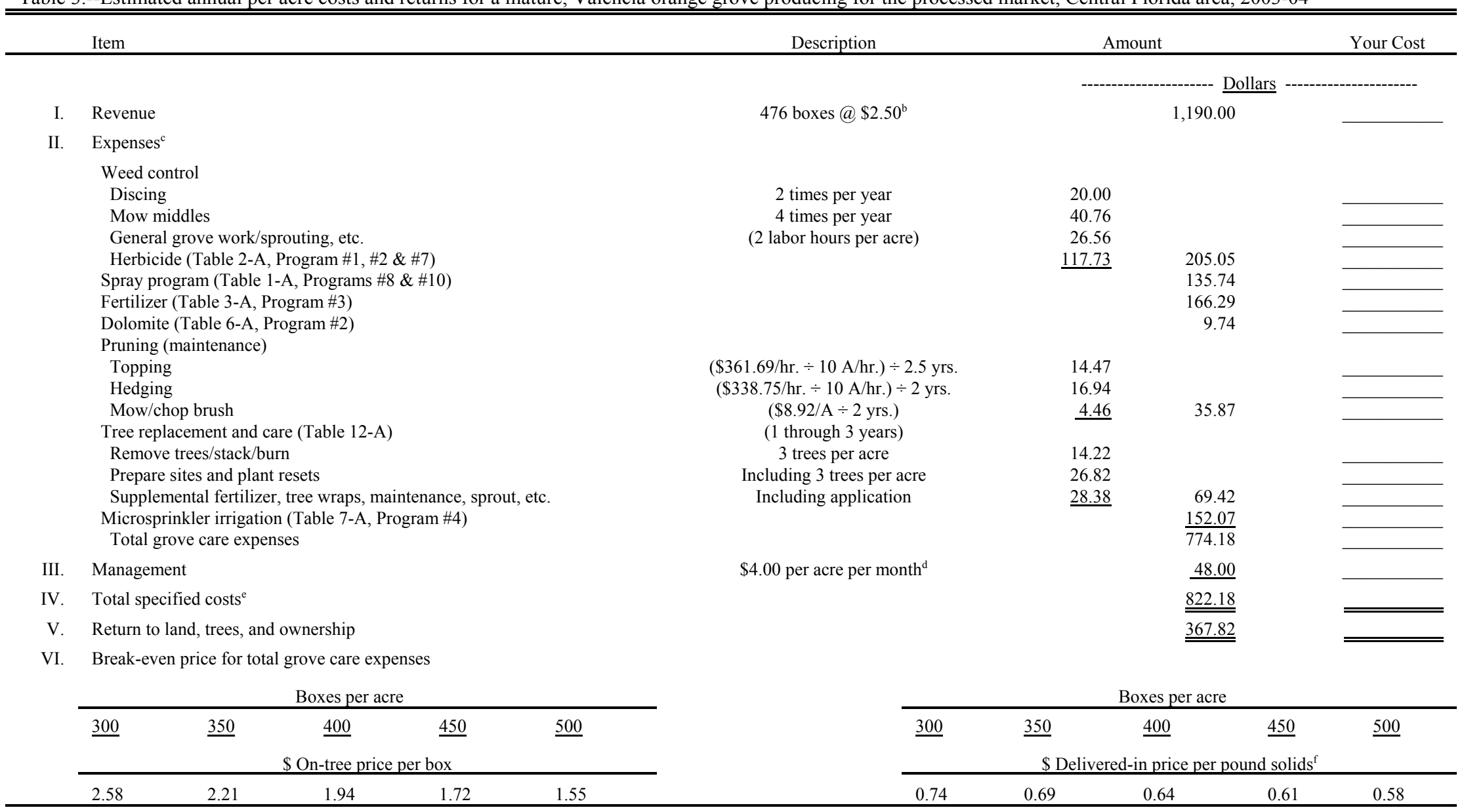

${ }^{a}$ While estimated annual per acre grove costs in Table 3 are representative for a mature Central Florida Valencia orange grove, grove care costs for specific grove site may differ depending on grove practices performed (e.g., Temik application would add $\$ 122.22$ per acre; extensive tree loss due to blight or tristeza would double tree replacement/care costs).

${ }^{\mathrm{b}}$ On-tree price per box is preliminary; assumes price for processed oranges only.

'Assumes material custom applied; therefore, a 10 percent handling and supervision charge is added to material cost.

${ }^{\mathrm{d}}$ Other methods to estimate a management cost (e.g., $5 \%$ of gross revenue or $10 \%$ of total grove care costs are used in the industry).

${ }^{e}$ Other cost items not included in budget are ad valorem taxes and interest on grove investment. In addition to these cost items, overhead and administrative costs (e.g., water drainage district taxes, crop insurance, and other grower assessments, can add up to $12 \%$ of total grove care costs). These costs vary from grove to grove.

${ }^{\mathrm{f}}$ Assumes 6.6 pounds solids per box and $\$ 2.19$ pick and haul cost per box (including canker decontamination costs) and Department of Citrus advertising assessment of $\$ 0.15$ per box. 
Table 4.--Estimated total delivered-in cost for Central Florida (Ridge) Valencia oranges grown for the processed market under three cultural cost programs, 2003-04

\begin{tabular}{|c|c|c|c|c|c|c|c|c|c|}
\hline \multirow[t]{2}{*}{$\begin{array}{l}\text { Represents a mature }(10+\text { years old) } \\
\text { Central Florida (Ridge) Orange Grove }\end{array}$} & \multicolumn{3}{|c|}{$\begin{array}{c}\text { Processed Valencia Orange } \\
\text { Low Cost Cultural Program } \\
\text { One-Year Alternative }\end{array}$} & \multicolumn{3}{|c|}{$\begin{array}{l}\text { Processed Valencia Orange } \\
\text { Cultural Program }\end{array}$} & \multicolumn{3}{|c|}{$\begin{array}{c}\text { Fresh/Processed Valencia Orange } \\
\text { Cultural Program }\end{array}$} \\
\hline & \$/Acre & \$/Box & \$/P.S. & $\$ /$ Acre & \$/Box & \$/P.S. & $\$ /$ Acre & $\$ /$ Box & \$/P.S. \\
\hline Total Production/Cultural Costs & $\$ 692.43$ & $\$ 1.455$ & $\$ 0.2204$ & $\$ 774.18$ & $\$ 1.626$ & $\$ 0.2464$ & $\$ 828.49$ & $\$ 1.741$ & $\$ 0.2637$ \\
\hline Interest on Operating (Cultural) Costs & 19.04 & 0.040 & 0.0061 & 21.29 & 0.045 & 0.0068 & 22.78 & 0.048 & 0.0073 \\
\hline Management Costs & 48.00 & 0.101 & 0.0153 & 48.00 & 0.101 & 0.0153 & 48.00 & 0.101 & 0.0153 \\
\hline \multicolumn{10}{|l|}{ Taxes/Regulatory Costs: } \\
\hline Property Tax and Water Management Tax & 61.87 & 0.130 & 0.0197 & 61.87 & 0.130 & 0.0197 & 61.87 & 0.130 & 0.0197 \\
\hline Canker Decontamination Costs & 5.52 & $\underline{0.012}$ & $\underline{0.0018}$ & 5.52 & $\underline{0.012}$ & $\underline{0.0018}$ & 5.52 & $\underline{0.012}$ & $\underline{0.0018}$ \\
\hline Total Direct Grower Costs & $\$ 826.86$ & $\$ 1.737$ & $\$ 0.2632$ & \$ 910.86 & $\$ 1.914$ & $\$ 0.2899$ & $\$ 966.66$ & $\$ 2.031$ & $\$ 0.3077$ \\
\hline Interest on Average Capital Investment Costs & 321.22 & $\underline{0.675}$ & $\$ 0.1022$ & 321.22 & $\underline{0.675}$ & $\underline{0.1022}$ & 321.22 & $\underline{0.675}$ & $\underline{0.1022}$ \\
\hline Total Grower Costs & $\$ 1,148.07$ & $\$ 2.412$ & $\$ 0.3654$ & $\$ 1,232.07$ & $\$ 2.588$ & $\$ 0.3922$ & $\$ 1,287.87$ & $\$ 2.706$ & $\$ 0.4099$ \\
\hline \multicolumn{10}{|l|}{ Harvesting and Assessment Costs: } \\
\hline \multicolumn{10}{|l|}{ Pick/Spot Pick, Roadside \& Haul and } \\
\hline Canker Decontamination Costs & $1,042.44$ & 2.190 & 0.3318 & $1,042.44$ & 2.190 & 0.3318 & $1,042.44$ & 2.190 & 0.3318 \\
\hline DOC Assessment & 71.40 & $\underline{0.150}$ & $\underline{0.0227}$ & 71.40 & $\underline{0.150}$ & $\underline{0.0227}$ & 71.40 & $\underline{0.150}$ & $\underline{0.0227}$ \\
\hline Total Harvesting and Assessment Costs & $1,113.84$ & 2.340 & 0.3545 & $1,113.84$ & 2.340 & 0.3545 & $1,113.84$ & 2.340 & 0.3545 \\
\hline Total Delivered-In Cost & $\$ \underline{\underline{2,261.91}}$ & $\$ \underline{\underline{4.752}}$ & $\$ \underline{\underline{0.7200}}$ & $\$ \underline{\underline{2,345.91}}$ & $\$ \underline{\underline{4.928}}$ & $\$ \underline{\underline{0.7467}}$ & $\$ \underline{\underline{2,401.71}}$ & $\$ \underline{\underline{\underline{5.046}}}$ & $\$ \underline{\underline{0.7645}}$ \\
\hline P.S. $=$ Pound Solids & \multicolumn{3}{|c|}{ Cultural program (Table 11-A) } & \multirow{2}{*}{\multicolumn{3}{|c|}{ Cultural program (Table 3) }} & \multirow{2}{*}{\multicolumn{3}{|c|}{$\begin{array}{l}\text { Cultural program (Table 11-A). } \\
\text { A Fall Miticide Spray added to cultura } \\
\text { program (Table 3) }\end{array}$}} \\
\hline $\begin{array}{l}\text { Yield: } 476 \text { boxes/acre @ 6.6 P.S. per box } \\
112 \text { trees per acre }\end{array}$ & \multicolumn{3}{|c|}{$\begin{array}{l}\text { Two summer oil sprays with oil, } \\
\text { copper, miticide and nutritionals }\end{array}$} & & & & & & \\
\hline
\end{tabular}


Table 5.--Estimated annual per acre costs and returns and 5-year average costs and returns for a mature, Valencia orange grove producing citrus for processing in the Central Florida area, 1999-00-2003-04

\begin{tabular}{|c|c|c|c|c|c|c|}
\hline Year & $\begin{array}{c}\text { On-tree } \\
\text { Price/Box } \\
\end{array}$ & Yield & $\begin{array}{c}\text { Gross } \\
\text { Revenue } \\
\end{array}$ & $\begin{array}{c}\text { Total Grove } \\
\text { Care Expenses } \\
\end{array}$ & $\begin{array}{c}\text { Total Ppecified } \\
\text { Costs }^{\mathrm{f}}\end{array}$ & $\begin{array}{l}\text { Net Return to Land, } \\
\text { Trees, and Ownership }\end{array}$ \\
\hline 1999-00 & $\$ 4.31$ & 448 & $1,930.88$ & 783.43 & 831.43 & $1,099.45$ \\
\hline $2000-01$ & $\$ 3.70$ & $436^{\mathrm{d}}$ & $1,613.20$ & $758.85^{\mathrm{e}}$ & 806.85 & 806.35 \\
\hline 2001-02 & $\$ 4.17$ & 446 & $1,859.82$ & 767.77 & 815.77 & $1,044.05$ \\
\hline $2002-03$ & $\$ 3.91$ & 446 & $1,743.86$ & 777.69 & 825.59 & 918.27 \\
\hline 2003-04 & $\$ 2.50^{\mathrm{b}}$ & $476^{\mathrm{c}}$ & $1,190.00$ & 774.18 & 822.18 & 367.82 \\
\hline
\end{tabular}

${ }^{a}$ On-tree prices for processed oranges only as reported by the Florida Agricultural Statistics Service.

${ }^{b}$ Preliminary estimate by authors at time of printing and is not a published price.

${ }^{c}$ Higher per acre yield is due to increased statewide production of Valencia oranges in 2003-04 season.

${ }^{\mathrm{d}}$ The severe drought affected yields for the 2000-01 season.

${ }^{\text {e} B e g a n ~ u s i n g ~ t w o ~ s u m m e r ~ o i l ~ s p r a y s ~(o n e ~ w i t h ~ n u t r i t i o n a l s) ~ i n ~ b u d g e t ~ e s t i m a t e s . ~}$

${ }_{\mathrm{f}}^{\mathrm{f}} \mathrm{A}$ management cost of $\$ 4.00$ per acre per month is included. Fixed costs such as taxes, debt service, and crop insurance are not included. 
Table 6.-- Estimated annual per acre costs and returns and 5-year average costs and returns (adjusted to 2004 dollars) for a mature, Valencia orange grove producing citrus for processing in the Central Florida area, 1999-00-2003-04

\begin{tabular}{|c|c|c|c|c|c|c|}
\hline Year & $\begin{array}{c}\text { Inflation } \\
\text { Factor Index }^{\mathrm{a}} \\
\end{array}$ & $\begin{array}{c}\text { Adjusted } \\
\text { On-tree } \\
\text { Price/Box } \\
\end{array}$ & Yield & $\begin{array}{c}\text { Gross } \\
\text { Revenue }\end{array}$ & $\begin{array}{c}\text { Total Specified } \\
\text { Costs }^{\mathrm{b}} \\
\end{array}$ & $\begin{array}{l}\text { Net Return to Land, } \\
\text { Trees, and Ownership }\end{array}$ \\
\hline & & & & 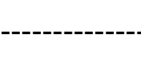 & Dollars & ------------ \\
\hline 1999-00 & 111.1 & $\$ 4.79$ & 448 & $2,145.92$ & 923.72 & $1,222.20$ \\
\hline 2001-02 & 112.4 & $\$ 4.69$ & 446 & $2,091.74$ & 916.93 & $1,174.81$ \\
\hline $2002-03$ & 106.7 & $\$ 4.17$ & 446 & $1,859.82$ & 880.90 & 978.92 \\
\hline 2003-04 & 100.0 & $\$ 2.50$ & 476 & $1,190.00$ & 822.18 & 367.82 \\
\hline 5-yr. avg. & - & $\$ 4.04$ & 450 & $1,818.00$ & 885.93 & 932.07 \\
\hline
\end{tabular}

${ }^{\text {a }}$ Producer price index for each year adjusted to 2004 prices $(2004=100)$, with 2004 producer price index estimated to be 147.4 . Producer price index for other years are: $2000=132.7 ; 2001=134.2 ; 2002=131.1 ;$ and $2003=138.1$.

${ }^{b}$ Management cost of $\$ 4.00$ per acre per month is included. Fixed costs such as taxes, debt service, and crop insurance are not included (Table 5). 


\section{REFERENCES}

1. Citrus Summary 2002-03. 2003. Florida Agricultural Statistics Service, Orlando, FL. http://www.nass.usda.gov/fl.

2. Muraro, Ronald P. 2004. A Listing of Estimated Comparative Central Florida (Ridge) Citrus Production Costs Per Acre for 2003-04. CREC Report, Citrus Research and Education Center, Lake Alfred, FL (July).

3. . 2004. Estimated Cost of Planting and Maintaining a Reset Citrus Tree through Three Years of Age. CREC Report, Citrus Research and Education Center, Lake Alfred, FL (July).

4. . 2004. A Listing of 2004 Custom Rates Reported by Twenty-Eight Ridge Citrus Caretakers. CREC Report, Citrus Research and Education Center, Lake Alfred, FL (July).

5. Savage, Zach. 1960. Citrus Yields Per Tree Age. UF Agricultural Extension Service Staff Paper SP60-8, University of Florida, Gainesville, FL.

6. Timmer, L.W., editor. 2004. 2004 Florida Citrus Pest Management Guide. Florida Cooperative Extension Service Staff Paper SP43, University of Florida, Gainesville, FL, 150pp (January).

7. Tucker, D. P.H., A.K. Alva, L.K. Jackson, and T.A. Wheaton (editors). 1995. Nutrition of Florida Citrus Trees. Florida Cooperative Extension Service Staff Paper SP169, University of Florida, Gainesville, FL, 61pp. 
ADDENDA: $\quad$ Listing of Grove Care Programs for Central Florida Citrus Production for Both Round Oranges and Grapefruit ${ }^{\mathrm{a}}$

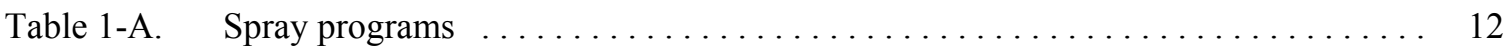

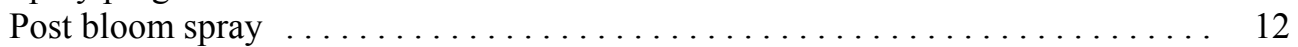

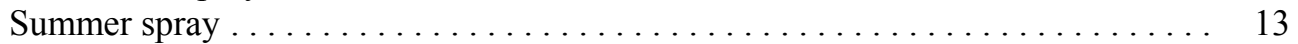

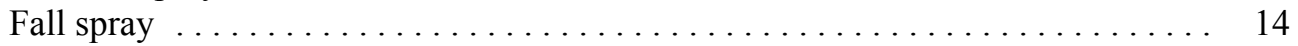

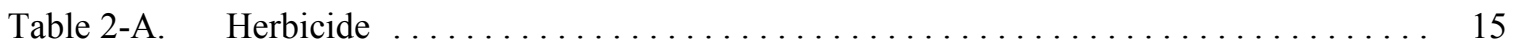

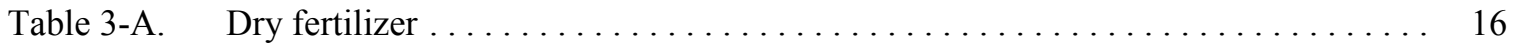

Table 4-A. Liquid fertilizer (double boom application) $\ldots \ldots \ldots \ldots \ldots \ldots \ldots \ldots \ldots \ldots \ldots$

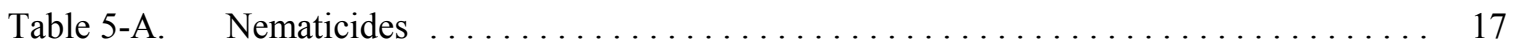

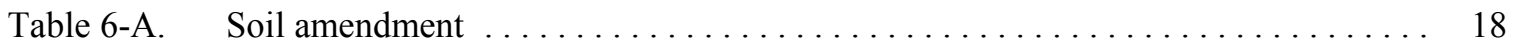

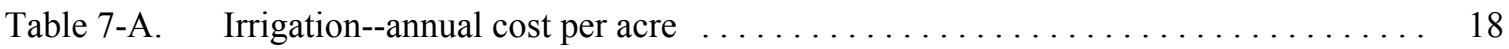

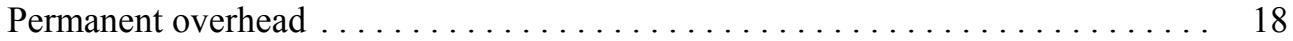

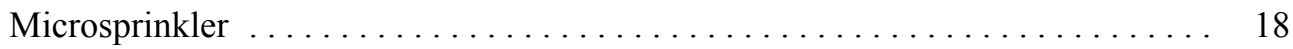

Table 8-A. A listing of 2004 custom rates reported by thirty-three Ridge citrus caretakers $\ldots \quad 19$

Table 9-A. $\quad 2004$ summary of average chemical price estimates $\ldots \ldots \ldots \ldots \ldots \ldots \ldots .21$

Table 10-A. 2004 summary of average fertilizer price estimates $\ldots \ldots \ldots \ldots \ldots \ldots \ldots \ldots$

Table 11-A. A listing of estimated comparative Central Florida (Ridge) citrus production

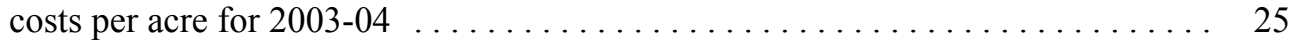

Table 12-A. Estimated cost of planting and maintaining a reset citrus tree through three years

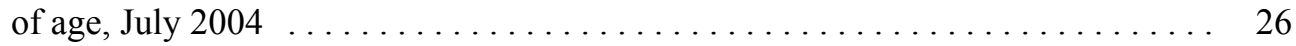

Table 13-A. Estimated average picking, roadsiding, and hauling charges for Florida citrus,

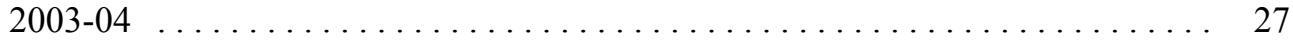

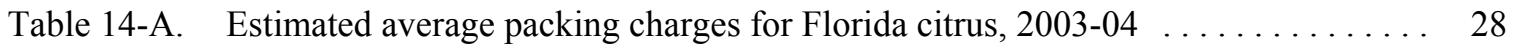

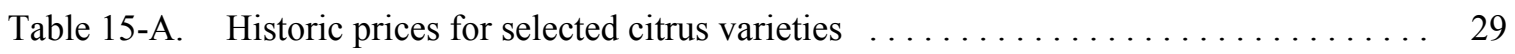

Table 16-A. Debt which can be supported per $\$ 1,000.00$ annual payment capacity $\ldots \ldots \ldots . \quad 30$

Abbreviations for important chemicals are:

$$
\begin{array}{llll}
\mathrm{B}=\text { Boron } & \mathrm{Fe}=\text { Iron } & \mathrm{Mn}=\text { Manganese } & \mathrm{Zn}=\text { Zinc } \\
\mathrm{Cu}=\text { Copper } & \mathrm{Mg}=\text { Magnesium } & \mathrm{N}=\text { Nitrogen } &
\end{array}
$$

${ }^{a}$ Costs in ADDENDA represent a custom managed operation. All equipment costs are based on average custom rate costs along with a 10 percent handling and supervision charge added to material cost. 
Table 1-A.--Spray programs

POST BLOOM SPRAY

\begin{tabular}{|c|c|c|c|c|}
\hline \multirow[t]{4}{*}{ Spray Program \#1 } & $\underline{\text { Materials/Ingredients }}$ & $\begin{array}{l}\text { Amount } \\
\text { /Acre }\end{array}$ & $\underline{\text { Cost/Acre }}$ & $\begin{array}{c}\text { Your } \\
\text { Cost/Acre } \\
\end{array}$ \\
\hline & $\begin{array}{l}\text { Oil } 97+\% \\
\mathrm{Cu}(50 \% \text { metallic }) \\
\mathrm{Zn} \\
\mathrm{Mn}\end{array}$ & $\begin{array}{l}5 \text { gallons } \\
10 \text { pounds } \\
5 \text { pounds } \\
10 \text { pounds }\end{array}$ & $\begin{array}{r}\$ 11.50 \\
13.20 \\
4.35 \\
3.40\end{array}$ & \\
\hline & $\begin{array}{l}\text { Ground Application } \\
\text { (PTO driven airblast) }\end{array}$ & 125 gallons & $\underline{22.95}$ & \\
\hline & Total per Application & & $\$ \underline{\underline{55.40}}$ & \\
\hline \multirow{4}{*}{$\begin{array}{l}\text { Spray Program \#2 } \\
\text { (Scab/melanose) }\end{array}$} & Materials/Ingredients & $\begin{array}{l}\text { Amount } \\
\text { /Acre }\end{array}$ & $\underline{\text { Cost/Acre }}$ & $\begin{array}{c}\text { Your } \\
\text { Cost/Acre } \\
\end{array}$ \\
\hline & $\begin{array}{l}\mathrm{Cu}(50 \% \text { metallic }) \\
\mathrm{Zn} \\
\mathrm{Mn} \\
\text { Micromite } 25 \mathrm{WP}\end{array}$ & $\begin{array}{l}10 \text { pounds } \\
5 \text { pounds } \\
10 \text { pounds } \\
1.25 \text { pounds }\end{array}$ & $\begin{array}{r}\$ 13.20 \\
4.35 \\
3.40 \\
39.88\end{array}$ & \\
\hline & $\begin{array}{l}\text { Ground Application } \\
\text { (PTO driven airblast) }\end{array}$ & 125 gallons & $\underline{22.95}$ & \\
\hline & Total per Application & & $\$ \underline{\underline{83.78}}$ & \\
\hline \multirow[t]{4}{*}{ Spray Program \#3 } & Materials/Ingredients & $\begin{array}{l}\text { Amount } \\
\text { /Acre } \\
\end{array}$ & $\underline{\text { Cost/Acre }}$ & $\begin{array}{c}\text { Your } \\
\text { Cost/Acre } \\
\end{array}$ \\
\hline & $\begin{array}{l}\mathrm{Cu}(50 \% \text { metallic }) \\
\text { Agri-Mek }\end{array}$ & $\begin{array}{l}15 \text { pounds } \\
10 \text { ounces }\end{array}$ & $\begin{array}{r}\$ 19.80 \\
45.38\end{array}$ & \\
\hline & $\begin{array}{l}\text { Ground Application } \\
\quad \text { (engine driven airblast) }\end{array}$ & 250 gallons & $\underline{29.67}$ & \\
\hline & Total per Application & & $\$ \underline{\underline{75.05}}$ & \\
\hline \multirow[t]{4}{*}{ Spray Program \#4 } & $\underline{\text { Materials/Ingredients }}$ & $\begin{array}{l}\text { Amount } \\
\text { /Acre } \\
\end{array}$ & $\underline{\text { Cost/Acre }}$ & $\begin{array}{c}\text { Your } \\
\text { Cost/Acre } \\
\end{array}$ \\
\hline & $\begin{array}{l}\text { Vendex 50WP } \\
\mathrm{Zn} \\
\mathrm{Mn}\end{array}$ & $\begin{array}{l}2 \text { pounds } \\
5 \text { pounds } \\
10 \text { pounds }\end{array}$ & $\begin{array}{r}\$ 31.36 \\
4.35 \\
3.40\end{array}$ & \\
\hline & $\begin{array}{l}\text { Ground Application } \\
\quad \text { (PTO driven airblast) }\end{array}$ & 125 gallons & $\underline{22.95}$ & \\
\hline & Total per Application & & $\$ \underline{\underline{62.06}}$ & \\
\hline
\end{tabular}


Table 1-A.--Spray programs (continued)

POST BLOOM SPRAY (continued)

\begin{tabular}{|c|c|c|c|c|}
\hline Spray Program \#5 & $\underline{\text { Materials/Ingredients }}$ & $\begin{array}{l}\text { Amount } \\
\text { /Acre } \\
\end{array}$ & $\underline{\text { Cost/Acre }}$ & $\begin{array}{c}\text { Your } \\
\text { Cost/Acre } \\
\end{array}$ \\
\hline \multirow[t]{3}{*}{ (Scale insects) } & Lorsban 4EC & 5 pints & $\$ 21.20$ & \\
\hline & $\begin{array}{l}\text { Ground Application } \\
\text { (engine driven airblast) }\end{array}$ & 500 gallons & $\underline{31.00}$ & \\
\hline & Total per Application & & $\$ 52.20$ & \\
\hline
\end{tabular}

\section{$\underline{\text { SUMMER SPRAY }}$}

\begin{tabular}{|c|c|c|c|c|}
\hline \multirow[t]{6}{*}{ Spray Program \#6 } & $\underline{\text { Materials/Ingredients }}$ & $\begin{array}{l}\text { Amount } \\
\text { /Acre }\end{array}$ & $\underline{\text { Cost/Acre }}$ & $\begin{array}{c}\text { Your } \\
\text { Cost/Acre } \\
\end{array}$ \\
\hline & Oil 97+\% & 5 gallons & $\$ 11.30$ & \\
\hline & $\mathrm{Cu}(50 \%$ material $)$ & 7 pounds & 9.24 & \\
\hline & Micro-Mite & 1.25 pounds & 39.88 & \\
\hline & $\begin{array}{l}\text { Ground Application } \\
\text { (PTO driven airblast) }\end{array}$ & 250 gallons & $\underline{29.67}$ & \\
\hline & Total per Application & & $\$ \underline{\underline{90.09}}$ & \\
\hline \multirow[t]{6}{*}{ Spray Program \#7 } & $\underline{\text { Materials/Ingredients }}$ & $\begin{array}{l}\text { Amount } \\
\text { /Acre } \\
\end{array}$ & $\underline{\text { Cost/Acre }}$ & $\begin{array}{c}\text { Your } \\
\text { Cost/Acre } \\
\end{array}$ \\
\hline & Oil $97+\%$ & 5 gallons & $\$ 11.30$ & \\
\hline & Agri-Mek & 10 ounces & 45.38 & \\
\hline & $\mathrm{Cu}(50 \%$ material $)$ & 7 pounds & 9.24 & \\
\hline & $\begin{array}{l}\text { Ground Application } \\
\quad \text { (engine driven airblast) }\end{array}$ & 250 gallons & $\underline{29.67}$ & \\
\hline & Total per Application & & $\$ \underline{\underline{95.59}}$ & \\
\hline \multirow[t]{6}{*}{ Spray Program \#8 } & $\underline{\text { Materials/Ingredients }}$ & $\begin{array}{l}\text { Amount } \\
\text { /Acre } \\
\end{array}$ & $\underline{\text { Cost/Acre }}$ & \multirow[t]{6}{*}{$\begin{array}{c}\text { Your } \\
\text { Cost/Acre }\end{array}$} \\
\hline & Oil 97+\% & 5 gallons & $\$ 11.30$ & \\
\hline & Micromite & 1.25 pounds & 39.88 & \\
\hline & $\mathrm{Cu}(50 \%$ material $)$ & 7 pounds & 9.24 & \\
\hline & $\begin{array}{l}\text { Ground Application } \\
\text { (PTO driven airblast) }\end{array}$ & 125 gallons & $\underline{22.95}$ & \\
\hline & Total per Application & & $\$ \underline{83.37}$ & \\
\hline
\end{tabular}


Table 1-A.--Spray programs (continued)

$\underline{\text { SUMMER SPRAY (continued) }}$

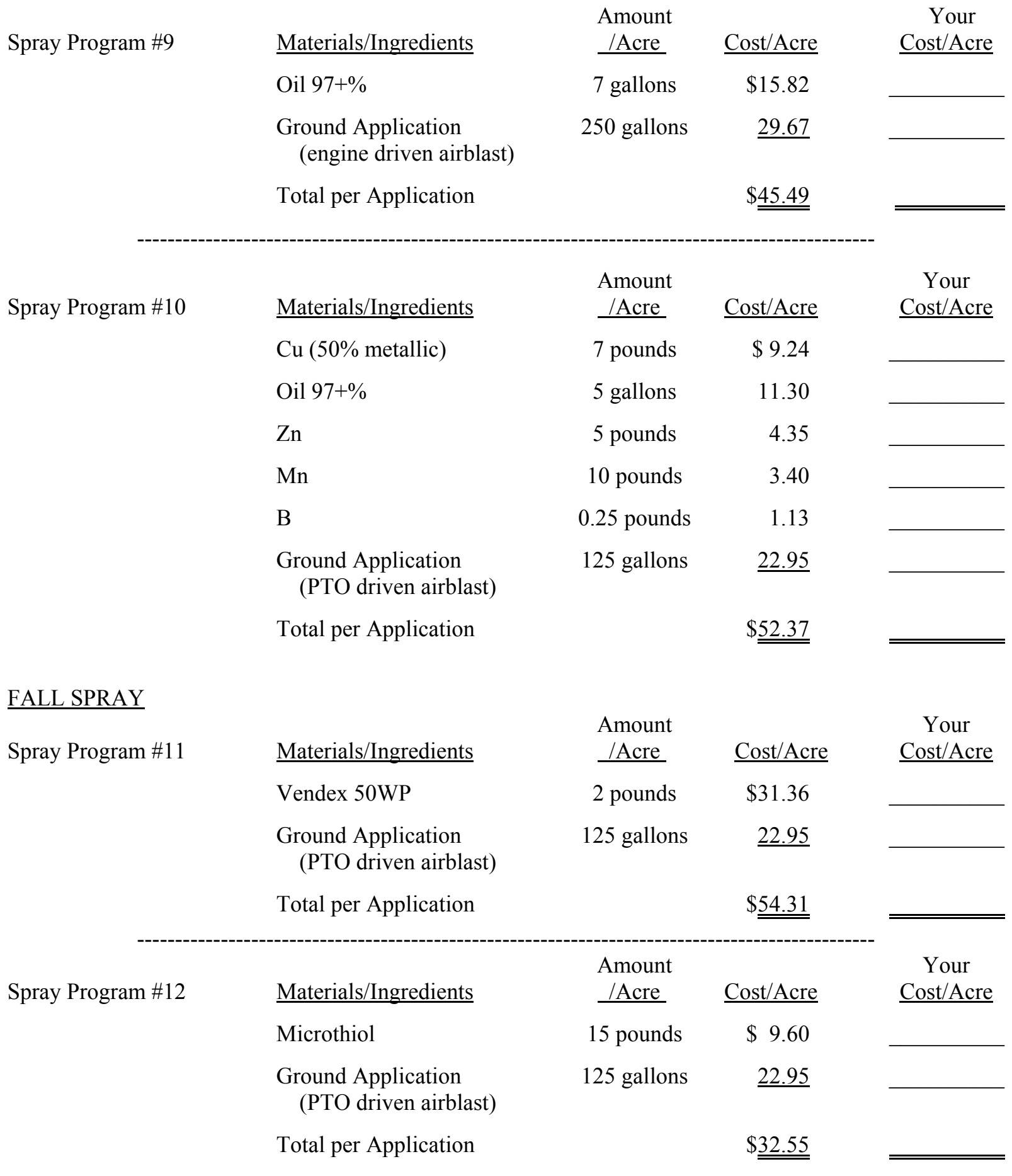


Table 2-A.--Herbicide

\begin{tabular}{|c|c|c|c|c|}
\hline \multirow{6}{*}{$\begin{array}{l}\text { Herbicide Program \#1 } \\
\text { (Strip/band) }\end{array}$} & $\underline{\text { Materials }}$ & $\begin{array}{c}\text { Amount/ } \\
\text { Treated Acre } \\
\end{array}$ & $\begin{array}{c}\text { Cost/ } \\
\text { Grove Acre }^{\mathrm{a}}\end{array}$ & $\begin{array}{r}\text { Your Cost/ } \\
\text { Grove Acre } \\
\end{array}$ \\
\hline & Solicam 80 DF & 3 pounds & $\$ 22.73$ & \\
\hline & Karmex WP & 4 pounds & 7.42 & \\
\hline & Roundup Ultra Max & 2 quarts & 8.08 & \\
\hline & $\begin{array}{l}\text { Ground Application } \\
\text { (1 time) }\end{array}$ & & $\underline{14.01}$ & \\
\hline & Total for 1 Application & & $\$ \underline{\underline{52.24}}$ & \\
\hline \multirow{6}{*}{$\begin{array}{l}\text { Herbicide Program \#2 } \\
\text { (Strip/band) }\end{array}$} & $\underline{\text { Materials }}$ & $\begin{array}{c}\text { Amount/ } \\
\text { Treated Acre }\end{array}$ & $\begin{array}{c}\text { Cost/ } \\
\text { Grove Acre }^{\mathrm{a}}\end{array}$ & $\begin{array}{r}\text { Your Cost/ } \\
\text { Grove Acre } \\
\end{array}$ \\
\hline & Mandate & 2 pints & $\$ 22.21$ & \\
\hline & Direx 4L & 3 quarts & 5.88 & \\
\hline & Roundup Ultra Max & 2 quarts & 8.08 & \\
\hline & $\begin{array}{l}\text { Ground Application } \\
\text { (1 time) }\end{array}$ & & $\underline{14.01}$ & \\
\hline & Total for 1 Application & & $\$ \underline{\underline{50.18}}$ & \\
\hline \multirow{5}{*}{$\begin{array}{l}\text { Herbicide Program \#3 } \\
\text { (Strip/band) }\end{array}$} & Materials & $\begin{array}{c}\text { Amount/ } \\
\text { Treated Acre }\end{array}$ & $\begin{array}{c}\text { Cost/ } \\
\text { Grove Acre }^{\mathrm{a}}\end{array}$ & $\begin{array}{r}\text { Your Cost/ } \\
\text { Grove Acre } \\
\end{array}$ \\
\hline & Karmex WP & 4 pounds & $\$ 7.42$ & \\
\hline & Roundup Ultra Max & 2 quarts & 8.08 & \\
\hline & $\begin{array}{l}\text { Ground Application } \\
\text { (1 time) }\end{array}$ & & $\underline{14.01}$ & \\
\hline & Total for 1 Application & & $\$ 29.51$ & \\
\hline \multirow{5}{*}{$\begin{array}{l}\text { Herbicide Program \#4 } \\
\text { (Strip/band) }\end{array}$} & $\underline{\text { Materials }}$ & $\begin{array}{c}\text { Amount/ } \\
\text { Treated Acre } \\
\end{array}$ & $\begin{array}{c}\text { Cost/ } \\
\text { Grove Acre }^{\mathrm{a}}\end{array}$ & $\begin{array}{r}\text { Your Cost/ } \\
\text { Grove Acre } \\
\end{array}$ \\
\hline & Roundup Ultra Max & 2 quarts & $\$ 8.08$ & \\
\hline & Ammonium Sulfate & 17 pounds & 1.39 & \\
\hline & $\begin{array}{l}\text { Ground Application } \\
\text { (1 time) }\end{array}$ & & $\underline{14.01}$ & \\
\hline & Total for 1 Application & & $\$ \underline{\underline{23.48}}$ & \\
\hline \multirow{5}{*}{$\begin{array}{l}\text { Herbicide Program \#5 } \\
\text { (Strip/band) }\end{array}$} & $\underline{\text { Materials }}$ & $\begin{array}{c}\text { Amount/ } \\
\text { Treated Acre }\end{array}$ & $\begin{array}{l}\text { Cost/ } \\
\text { Grove Acre }^{\mathrm{a}}\end{array}$ & $\begin{array}{r}\text { Your Cost/ } \\
\text { Grove Acre } \\
\end{array}$ \\
\hline & Roundup Ultra Max & 2 quarts & $\$ 8.08$ & \\
\hline & Princep (Caliber 90) & 4 pounds & 6.72 & \\
\hline & $\begin{array}{l}\text { Ground Application } \\
\text { (1 time) }\end{array}$ & & $\underline{14.01}$ & \\
\hline & Total for 1 Application & & $\$ 28.81$ & \\
\hline
\end{tabular}


Table 2-A.--Herbicide (continued)

\begin{tabular}{|c|c|c|c|c|}
\hline \multirow{6}{*}{$\begin{array}{l}\text { Herbicide Program \#6 } \\
\text { (Strip/band) }\end{array}$} & $\underline{\text { Materials }}$ & $\underline{\text { Treated Acre }}$ & $\underline{\text { Grove Acre }}^{\mathrm{a}}$ & Grove Acre \\
\hline & Direx 4L & 3 quarts & $\$ 5.88$ & \\
\hline & Solicam & 3 pounds & 22.73 & \\
\hline & Roundup Ultra Max & 2 quarts & 8.08 & \\
\hline & $\begin{array}{l}\text { Ground Application } \\
\text { (1 time) }\end{array}$ & & $\underline{14.01}$ & \\
\hline & Total for 1 Application & & $\$ \underline{\underline{50.70}}$ & \\
\hline \multirow{4}{*}{$\begin{array}{l}\text { Herbicide Program \#7 } \\
\text { (Spot herbicide for } \\
\text { grass/brush regrowth } \\
\text { under trees.) }\end{array}$} & Materials & $\begin{array}{c}\text { Amount/ } \\
\text { Treated Acre }\end{array}$ & $\begin{array}{c}\text { Cost/ } \\
\text { Grove Acre }^{\mathrm{a}}\end{array}$ & $\begin{array}{r}\text { Your Cost/ } \\
\text { Grove Acre }\end{array}$ \\
\hline & Roundup Ultra Max & 2 quarts & $\$ 8.08$ & \\
\hline & $\begin{array}{l}\text { Ground Application } \\
\text { (1 time) }\end{array}$ & 15 gallons & 7.23 & \\
\hline & Total for 1 Application & & $\$ 15.31$ & \\
\hline
\end{tabular}

${ }^{a}$ For herbicide materials, amount per grove acre does not equal amount per treated acre shown on label, only a strip or band is being treated. This report assumes that only half a grove surface is being treated.

Table 3-A. Dry Fertilizer

\begin{tabular}{|c|c|c|c|c|}
\hline \multirow{4}{*}{$\begin{array}{l}\text { Program \#1 } \\
(162 \mathrm{lbs} \text { N/Acre })\end{array}$} & $\begin{array}{l}\text { Analysis/Material } \\
\text { Applied } \\
\end{array}$ & $\begin{array}{l}\text { Amount } \\
\text { /Acre }\end{array}$ & $\underline{\text { Cost/Acre }}$ & $\begin{array}{c}\text { Your } \\
\text { Cost/Acre } \\
\end{array}$ \\
\hline & $12-2-12-2.4 \mathrm{MgO}$ & 1350 pounds & $\$ 130.82$ & \\
\hline & Application & 3 times & $\underline{26.04}$ & \\
\hline & Total for 3 Applications & & $\$ \underline{\underline{156.86}}$ & \\
\hline \multirow{4}{*}{$\begin{array}{l}\text { Program \#2 } \\
\text { (180 lbs N/Acre) }\end{array}$} & $\begin{array}{l}\text { Analysis/Material } \\
\text { Applied }\end{array}$ & $\begin{array}{l}\text { Amount } \\
\text { /Acre }\end{array}$ & $\underline{\text { Cost/Acre }}$ & $\begin{array}{c}\text { Your } \\
\text { Cost/Acre } \\
\end{array}$ \\
\hline & $16-0-16-4 \mathrm{MgO}$ & 1125 pounds & $\$ 123.75$ & \\
\hline & Application & 3 times & $\underline{26.04}$ & \\
\hline & Total for 3 Applications & & $\$ \underline{\underline{149.79}}$ & \\
\hline \multirow{4}{*}{$\begin{array}{l}\text { Program \#3 } \\
\text { (204 lbs N/Acre) }\end{array}$} & $\begin{array}{l}\text { Analysis/Material } \\
\text { Applied }\end{array}$ & $\begin{array}{l}\text { Amount } \\
\text { /Acre }\end{array}$ & $\underline{\text { Cost/Acre }}$ & $\begin{array}{c}\text { Your } \\
\text { Cost/Acre } \\
\end{array}$ \\
\hline & $16-0-16-4 \mathrm{MgO}$ & 1275 pounds & $\$ 140.25$ & \\
\hline & Application & 3 times & $\underline{26.04}$ & \\
\hline & Total for 3 Applications & & $\$ \underline{\underline{166.29}}$ & \\
\hline \multirow{4}{*}{$\begin{array}{l}\text { Program \#4 } \\
(225 \mathrm{lbs} \text { N/Acre })\end{array}$} & $\begin{array}{l}\text { Analysis/Material } \\
\text { Applied } \\
\end{array}$ & $\begin{array}{l}\text { Amount } \\
\text { /Acre }\end{array}$ & $\underline{\text { Cost/Acre }}$ & $\begin{array}{c}\text { Your } \\
\text { Cost/Acre } \\
\end{array}$ \\
\hline & $15-2-15-2.4 \mathrm{MgO}$ & 1500 pounds & $\$ 151.50$ & \\
\hline & Application & 3 times & $\underline{26.04}$ & \\
\hline & Total for 3 Applications & & $\$ 177.54$ & \\
\hline
\end{tabular}


Table 4-A.--Liquid Fertilizer (double boom application)

\begin{tabular}{|c|c|c|c|c|}
\hline Program \#1 & $\begin{array}{l}\text { Analysis/Material } \\
\text { Applied } \\
\end{array}$ & $\begin{array}{l}\text { Amount } \\
\text { /Acre }\end{array}$ & $\underline{\text { Cost/Acre }}$ & $\begin{array}{c}\text { Your } \\
\text { Cost/Acre } \\
\end{array}$ \\
\hline \multirow[t]{3}{*}{ (180 lbs N/Acre) } & $10-0-10$ & 1800 pounds & $\$ 138.42$ & \\
\hline & Double Boom Application & 3 times & 45.99 & \\
\hline & Total for 3 Applications & & $\$ \underline{\underline{184.41}}$ & \\
\hline \multirow{4}{*}{$\begin{array}{l}\text { Program \#2 } \\
(180 \mathrm{lbs} \text { N/Acre })\end{array}$} & $\begin{array}{l}\text { Analysis/Material } \\
\text { Applied } \\
\end{array}$ & $\begin{array}{l}\text { Amount } \\
\text { /Acre }\end{array}$ & $\underline{\text { Cost/Acre }}$ & $\begin{array}{c}\text { Your } \\
\text { Cost/Acre } \\
\end{array}$ \\
\hline & $10-2-10$ & 1800 pounds & $\$ 140.22$ & \\
\hline & Double Boom Application & 3 times & $\underline{45.99}$ & \\
\hline & Total for 3 Applications & & $\$ \underline{\underline{186.21}}$ & \\
\hline \multirow{6}{*}{$\begin{array}{l}\text { Program \#3 } \\
(180 \mathrm{lbs} \text { N/Acre })\end{array}$} & $\begin{array}{l}\text { Analysis/Material } \\
\text { Applied }\end{array}$ & $\begin{array}{l}\text { Amount } \\
\text { /Acre }\end{array}$ & $\underline{\text { Cost/Acre }}$ & $\begin{array}{c}\text { Your } \\
\text { Cost/Acre } \\
\end{array}$ \\
\hline & $10-0-10$ & 1800 pounds & $\$ 138.42$ & \\
\hline & Solicam 80 DF & 3 pounds* & 22.73 & \\
\hline & Karmex WP & 4 pounds* & 7.42 & \\
\hline & Double Boom Application & 3 times & $\underline{45.99}$ & \\
\hline & Total for 3 Applications & & $\$ \underline{\underline{214.56}}$ & \\
\hline
\end{tabular}

Table 5-A.--Nematicides

\begin{tabular}{|c|c|c|c|c|}
\hline \multirow[t]{4}{*}{ Program \#1 } & $\begin{array}{l}\text { Analysis/Material } \\
\text { Applied } \\
\end{array}$ & $\begin{array}{l}\text { Amount } \\
\text { /Acre }\end{array}$ & $\underline{\text { Cost/Acre }}$ & $\begin{array}{c}\text { Your } \\
\text { Cost/Acre } \\
\end{array}$ \\
\hline & Temik 15G & 33 pounds & $\$ 107.58$ & \\
\hline & Application & & 14.64 & \\
\hline & Total per Application & & $\$ \underline{122.22}$ & \\
\hline \multirow[t]{4}{*}{ Program \#2 } & $\begin{array}{l}\text { Analysis/Material } \\
\text { Applied }\end{array}$ & $\begin{array}{l}\text { Amount } \\
\text { /Acre }\end{array}$ & $\underline{\text { Cost/Acre }}$ & $\begin{array}{c}\text { Your } \\
\text { Cost/Acre } \\
\end{array}$ \\
\hline & Temik 15G & 17 pounds & $\$ 55.42$ & \\
\hline & Application & & 14.64 & \\
\hline & Total per Application & & $\$ 70.06$ & \\
\hline
\end{tabular}


Table 6-A.--Soil Amendment

\begin{tabular}{|c|c|c|c|c|}
\hline Program \#1 & $\begin{array}{l}\text { Analysis/Material } \\
\text { Applied }\end{array}$ & $\begin{array}{l}\text { Amount } \\
\text { /Acre } \\
\end{array}$ & $\underline{\text { Cost/Acre }}$ & $\begin{array}{c}\text { Your } \\
\text { Cost/Acre } \\
\end{array}$ \\
\hline \multirow[t]{4}{*}{ (Every 3 years) } & Dolomite (Delivered) & 1 ton & $\$ 30.27$ & \\
\hline & Application & 1 time & 8.68 & \\
\hline & Total for 1 Application & & $\$ \underline{\underline{38.95}}$ & \\
\hline & (Average $1 / 3$ Ton Applied/Yr) & & $\$ \underline{\underline{12.98}}$ & \\
\hline \multirow{5}{*}{$\begin{array}{l}\text { Program \#2 } \\
\text { (Every } 4 \text { years) }\end{array}$} & $\begin{array}{l}\text { Analysis/Material } \\
\text { Applied } \\
\end{array}$ & $\begin{array}{l}\text { Amount } \\
\text { /Acre }\end{array}$ & $\underline{\text { Cost/Acre }}$ & $\begin{array}{c}\text { Your } \\
\text { Cost/Acre } \\
\end{array}$ \\
\hline & Dolomite (Delivered) & 1 ton & $\$ 30.27$ & \\
\hline & Application & 1 time & 8.68 & \\
\hline & Total for 1 Application & & $\$ \underline{\underline{38.95}}$ & \\
\hline & (Average 1/4 Ton Applied/Yr) & & $\$ 9.74$ & \\
\hline
\end{tabular}

Table 7-A.--Irrigation (annual cost per acre)

PERMANENT OVERHEAD

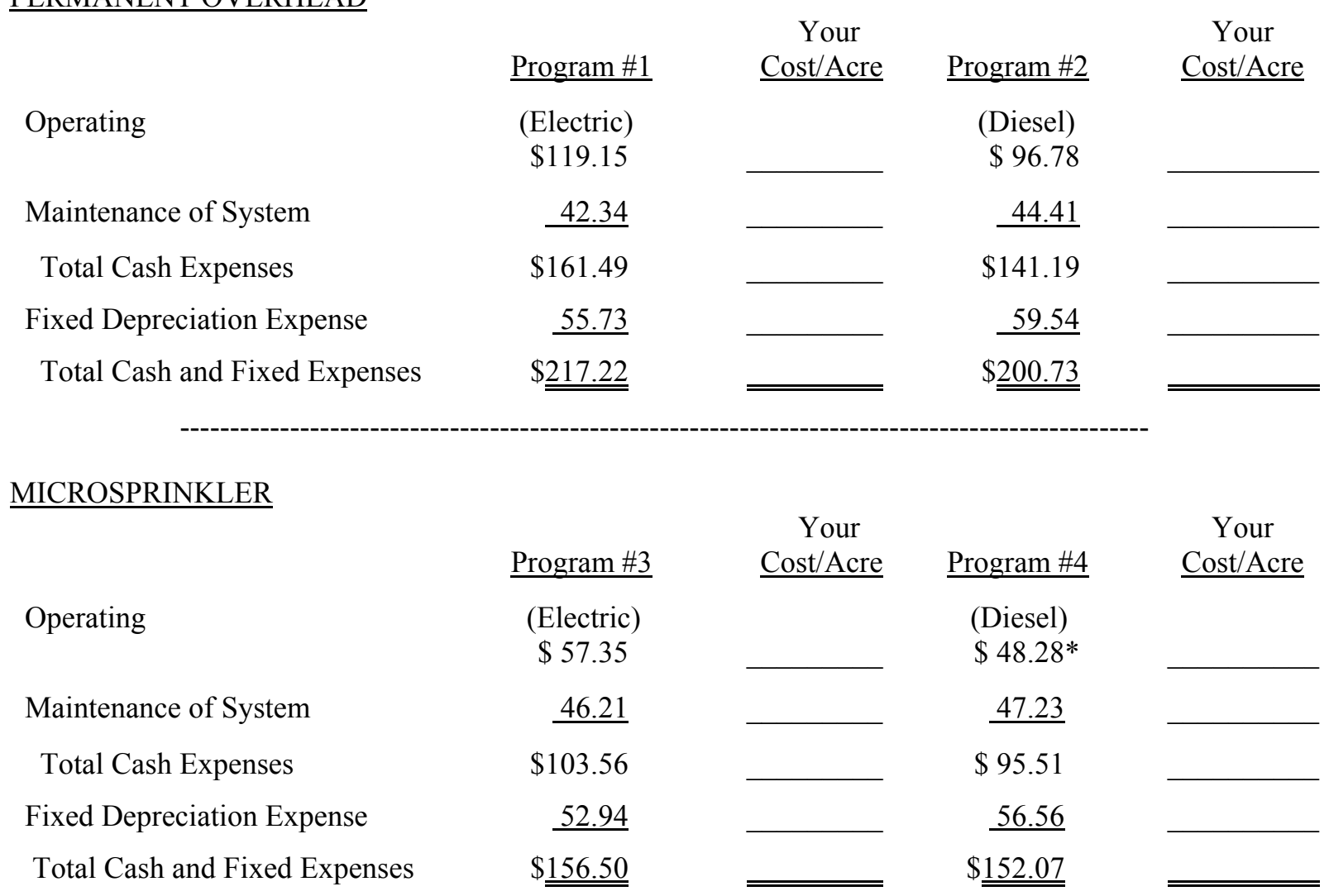

*Reflects the higher cost of fuel; diesel and electricity. 
19

Table 8-A.--A listing of 2004 custom rates reported by twenty-eight Ridge citrus caretakers

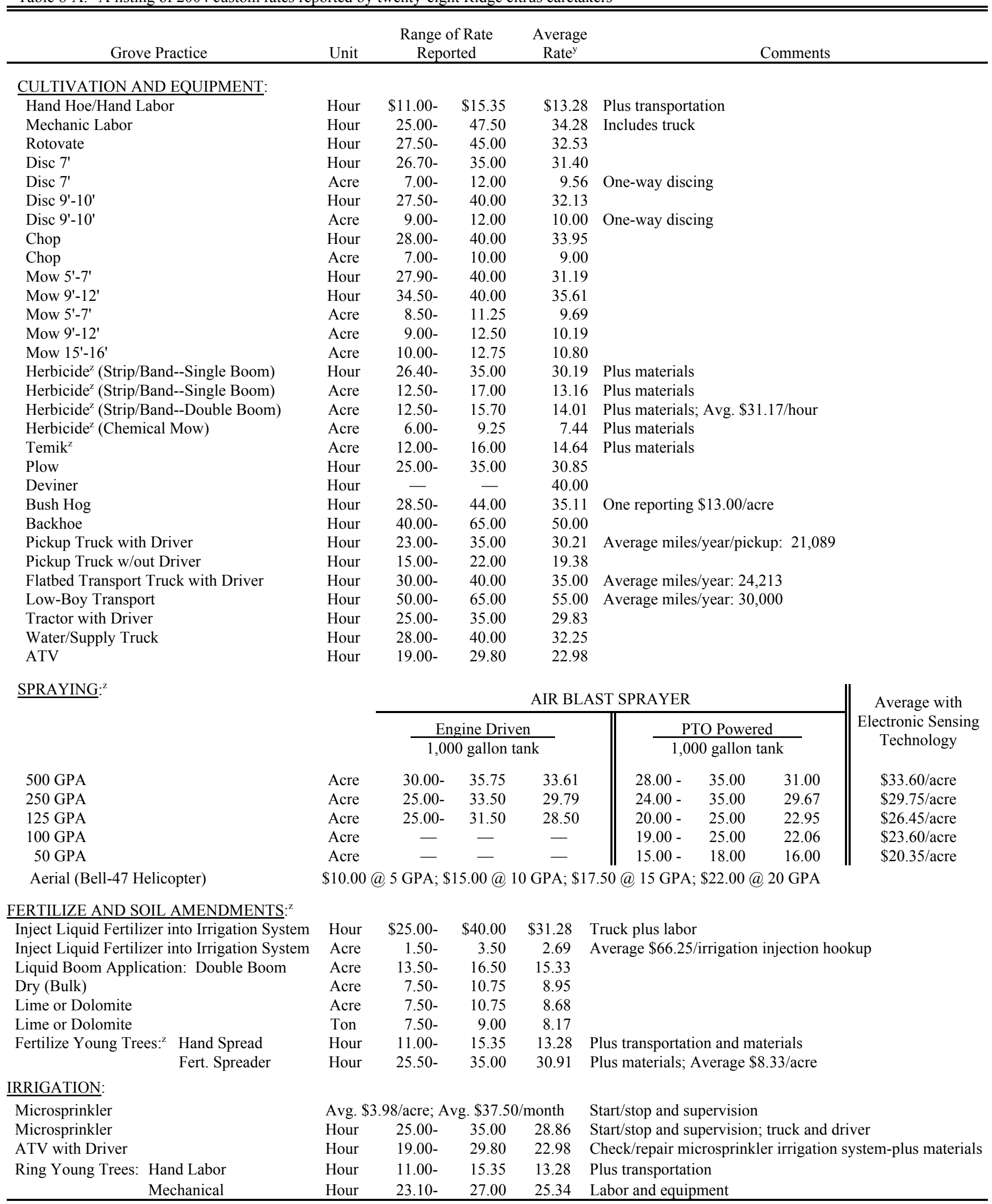


Table 8-A.--A listing of 2004 custom rates reported by twenty-eight Ridge citrus caretakers (continued)

\begin{tabular}{|c|c|c|c|c|c|}
\hline Grove Practice & Unit & \multicolumn{2}{|c|}{$\begin{array}{c}\text { Range of Rate } \\
\text { Reported }\end{array}$} & $\begin{array}{l}\text { Average } \\
\text { Rate }^{\mathrm{y}}\end{array}$ & \multirow[t]{2}{*}{ Comments } \\
\hline \multicolumn{5}{|l|}{ REMOVING TREES: } & \\
\hline Tree Shearing (Cutting Tree at Ground Level) & Hour & $\$ 50.00-$ & $\$ 65.00$ & $\$ 59.13$ & Average trees sheared: 5 to 20 trees/hour \\
\hline Front-end Loader & Hour & $50.00-$ & 60.00 & 54.02 & Average trees removed: 5 to 15 trees/hour \\
\hline Bulldozer & Hour & - & - & 50.00 & \\
\hline Front-end Loader with Tree Spade & Hour & - & - & 65.00 & \\
\hline \multicolumn{6}{|l|}{ PRUNING: } \\
\hline$\overline{\text { Power Saw with Operator }}$ & Hour & $\$ 17.00-$ & $\$ 25.00$ & $\$ 19.94$ & Plus transportation \\
\hline Limb Lifter/Tree Skirt Trimmer (Double Sided) & Hour & - & - & 205.00 & Cover $9-18$ acres one pass \\
\hline \multicolumn{6}{|l|}{ Hedging: } \\
\hline Single Side (Tractor Mounted) & Hour & $75.00-$ & 80.00 & 76.67 & Cover 2-5 acres/hour \\
\hline Double Side (Tractor Pulled) & Hour & $80.00-$ & 100.00 & 88.96 & Cover 3-5 acres/hour \\
\hline Double Side (Tractor Mounted) & Hour & - & - & 250.00 & \\
\hline Double Side (Self Propelled) ${ }^{\mathrm{x}}$ & Hour & $325.00-$ & 360.00 & 338.75 & Cover $10-25$ acres/hour depending on wood size \\
\hline Double Side (Self Propelled) ${ }^{\mathrm{x}}$ & Hour & $250.00-$ & 300.00 & 277.50 & Cover 4-12 acres/hour depending on wood size \\
\hline \multicolumn{6}{|l|}{ Topping: } \\
\hline Tractor Mounted & Hour & $195.00-$ & 200.00 & 196.67 & \\
\hline Tractor Pulled & Hour & - & - & 100.00 & Cover $1-3$ acres/hour \\
\hline Self Propelled & Hour & $345.00-$ & 375.00 & 361.69 & Cover 5-10 acres/hr (Roof Top); 5-20 acres/hr (Flat Top) \\
\hline Double Boom (Self Propelled) & Hour & - & - & 550.00 & Cover 2-12 acres/hr (Roof Top);15-30 acres/hr (Flat Top) \\
\hline \multicolumn{6}{|l|}{ Removing Brush: } \\
\hline Haul Brush out of Grove & Hour & $29.00-$ & 40.00 & 35.17 & Tractor-trailer/truck, driver plus 1 person; plus 2 people \\
\hline Front-end Loader (Push Brush) & Hour & $50.00-$ & 60.00 & 53.56 & $2-10$ acres/hour \\
\hline Chop/Mow Brush & Hour & $30.00-$ & 37.50 & 33.95 & 3-6 acres/hour; Average \$11.67/acre \\
\hline \multicolumn{6}{|l|}{ COLD PROTECTION: } \\
\hline$\overline{\text { Mechanical (Bank and Unbank) }}$ & Hour & $\$-$ & $\$-$ & $\$ 23.43$ & \\
\hline Install Wraps & Each & $0.15-$ & 0.50 & 0.36 & \\
\hline Cost Wraps & Each & $0.30-$ & 0.50 & 0.41 & \\
\hline \multicolumn{6}{|l|}{ OTHER CUSTOM RATES: } \\
\hline Plant Resets & Per Tree & $\$ 2.00-$ & $\$ 4.00$ & $\$ 2.50$ & Stake, plant and first watering \\
\hline Solid Set Planting & Per Tree & $1.00-$ & 1.50 & 1.33 & Stake, plant and first watering \\
\hline Travel/Setup Charge & Hour & - & - & 30.00 & Average for those reporting; One reporting $\$ 75.00$ \\
\hline \multicolumn{6}{|l|}{ Grove Management Charge/Month: } \\
\hline Supervising Grove Care Operations & Acre & $2.00-$ & 5.50 & 3.15 & \multirow{3}{*}{$\begin{array}{r}\text { In addition to caretaking charges; One reporting } 6 \% \text { of } \\
\text { lequipment labor charge }\end{array}$} \\
\hline Handling Fruit Marketing & Box & $0.10-$ & 0.25 & 0.15 & \\
\hline Supervising/Handling Chemicals/Fertilizer & $10 \%$ to 25 & $\%$ of mater & als cost & & \\
\hline $\begin{array}{l}\text { Charge for personnel to oversee harvesting } \\
\text { operations and coordinate harvest in } \\
\text { different blocks/groves and keeping of } \\
\text { harvesting labor compliance record. }\end{array}$ & \multicolumn{4}{|c|}{$10 \notin /$ box to $18 \notin /$ box; average $16 \phi /$ box } & $\begin{array}{l}\text { Note: } \\
\text { One reporting adding a } 5 \% \text { fuel surcharge if } \\
\text { gasoline price is above } \$ 1.50 / \text { gallon and diesel } \\
\text { fuel above } \$ 1.00 / \text { gallon. }\end{array}$ \\
\hline Consulting & \multicolumn{5}{|c|}{$\begin{array}{l}\text { Cultural Management/Horticultural Evaluation - } \$ 50 / \mathrm{hr} \text { to } \$ 200 / \mathrm{hr} \\
\text { Financial Analysis Prospectus - } \$ 350 / \mathrm{hr}\end{array}$} \\
\hline \multicolumn{6}{|l|}{ Total Reported Acreage Provided Grove } \\
\hline Service to: & Acre & $500-$ & 13,872 & 3,342 & Total acres reporting: 63,498 \\
\hline
\end{tabular}

${ }^{z}$ Plus materials. Caretakers reporting rates include labor, tractor and sprayer; supply truck included by most caretakers.

${ }^{y}$ Calculated by dividing the total number of caretakers reporting a grove practice rate into the sum reported. Unless otherwise stated, labor included with all charges.

${ }^{x}$ Low acres is for 2-year regrowth hedging; high acres is for annual maintenance hedging.

Source: Ronald P. Muraro, Extension Farm Management Economist, Lake Alfred CREC, July 2004. 
Table 9-A.--2004 summary of average chemical price estimates

\begin{tabular}{|c|c|c|c|c|}
\hline \multicolumn{2}{|l|}{ Item } & \multirow{2}{*}{$\frac{\text { Unit }}{\text { gallon }}$} & \multirow{2}{*}{$\begin{array}{c}\begin{array}{c}\text { Average } \\
\text { Price }\end{array} \\
196.38\end{array}$} & \multirow[t]{2}{*}{$\begin{array}{c}\text { Your Price } \\
(2004)\end{array}$} \\
\hline Fungicides: & Abound EC & & & \\
\hline & Aliette 80WP & pound & 10.16 & \\
\hline & Basic Copper Sulfate & pound & 1.20 & \\
\hline & Copper Hydroxide & pound & & \\
\hline & Copper (Kocide 101) & pound & 1.58 & \\
\hline & Copper (Kocide 2000) & pound & 2.07 & \\
\hline & Copper (Champ II Flowable) & gallon & 21.80 & \\
\hline & Cuprofix Disperss & pound & 1.79 & \\
\hline & Nu-Cop 50 DF & pound & 1.78 & \\
\hline & Enable & gallon & 51.60 & \\
\hline & Gem 25 & 40 ozs. & 103.33 & \\
\hline & Headline EC & gallon & 182.52 & \\
\hline & Oil - 435 or 455 & gallon & 2.05 & \\
\hline & Oil - 470 (Bio-lever) & gallon & 2.35 & \\
\hline & Ridomil Gold EC & gallon & 591.67 & \\
\hline & Topsin & pound & 13.40 & \\
\hline \multicolumn{5}{|c|}{ Insecticides/Nematicides: } \\
\hline & Admire $2 \mathrm{~F}$ & gallon & 454.00 & \\
\hline & Agri-Mek (0.15EC) & gallon & 526.67 & \\
\hline & Carbaryl 4L & gallon & 26.55 & \\
\hline & Carbaryl 80S & pound & 4.46 & \\
\hline & Chlorpyrifos $4 \mathrm{E}$ & gallon & 50.18 & \\
\hline & Clinch Fire Ant Bait & pound & 8.63 & \\
\hline & Danitol & gallon & 129.38 & \\
\hline & Guthion 2L & gallon & 29.96 & \\
\hline & Guthion 50WP & pound & 8.19 & \\
\hline & Imidan 70W (Diaprepes) & pound & 7.50 & \\
\hline & Lorsban 4EC & gallon & 30.82 & \\
\hline & Lorsban $15 \mathrm{G}$ & pound & 1.74 & \\
\hline & Malathion 5 EC & gallon & 21.66 & \\
\hline & Micromite $25 \mathrm{WS}$ & pound & 29.00 & \\
\hline & Micromite $80 \mathrm{WG}$ & gallon & 82.25 & \\
\hline & Microthiol & pound & 0.58 & \\
\hline & Nexter 75WP & pound & 85.16 & \\
\hline & Sevin $80 \mathrm{~S}$ & pound & 4.59 & \\
\hline & Sevin XLR & gallon & 26.62 & \\
\hline & Sulphur 6F & gallon & 3.10 & \\
\hline & Temik 15G & pound & 2.96 & \\
\hline & Vendex 50W & pound & 14.25 & \\
\hline
\end{tabular}


Table 9-A.--2004 summary of average chemical price estimates (continued)

\begin{tabular}{|c|c|c|c|c|}
\hline \multicolumn{2}{|l|}{ Item } & \multirow{2}{*}{$\frac{\text { Unit }}{\text { gallon }}$} & \multirow{2}{*}{$\begin{array}{c}\begin{array}{c}\text { Average } \\
\text { Price }\end{array} \\
42.53\end{array}$} & \multirow[t]{2}{*}{$\begin{array}{c}\text { Your Price } \\
(2004)\end{array}$} \\
\hline Herbicides: & Aqua Master & & & \\
\hline & Diuron 4L & gallon & 16.13 & \\
\hline & Direx 4L & gallon & 14.23 & \\
\hline & Direx $80 \mathrm{DF}$ & pound & 3.06 & \\
\hline & Fusilade DX 2E & gallon & 117.67 & \\
\hline & \multicolumn{4}{|l|}{ Glyphosate: } \\
\hline & Glyphomax Plus & gallon & 15.25 & \\
\hline & Roundup (Original) & gallon & 22.25 & \\
\hline & Roundup - Ultra Max & gallon & 29.37 & \\
\hline & Touchdown & gallon & 33.17 & \\
\hline & Gramoxone E (Paraquat) & gallon & 34.92 & \\
\hline & Hyvar X $80 \mathrm{WP}$ & pound & 16.98 & \\
\hline & Karmex $80 \mathrm{DF}$ & pound & 3.37 & \\
\hline & Krovar I & pound & 10.10 & \\
\hline & Landmaster II & gallon & 17.39 & \\
\hline & Mandate 2E & gallon & 161.53 & \\
\hline & Pendimax & gallon & 22.73 & \\
\hline & Poast Plus 1.0 EC & gallon & 52.39 & \\
\hline & Princep (Caliber 90) & pound & 3.05 & \\
\hline & Princep 4L & gallon & 13.22 & \\
\hline & Prowl & gallon & 21.48 & \\
\hline & Simazine $90 \mathrm{DF}$ & pound & 2.66 & \\
\hline & Simazine 4L & gallon & 12.87 & \\
\hline & Solicam $80 \mathrm{DF}$ & pound & 13.77 & \\
\hline & Simtrol & & 18.00 & \\
\hline & Surflan & gallon & 76.53 & \\
\hline \multicolumn{5}{|c|}{ Growth Regulators: } \\
\hline & Citrus Fix & gallon & 457.00 & \\
\hline & Pro-Gibb 3.91\% & 20-ounce bottle & 30.79 & \\
\hline & Tree-Hold & gallon & 79.17 & \\
\hline \multicolumn{5}{|c|}{ Other Spray Materials: } \\
\hline & Borates $(15 \%)$ & pound & 0.68 & \\
\hline & Manganese $(32 \%)$ & pound & 0.31 & \\
\hline & Zinc $(78 \%)$ & pound & 0.79 & \\
\hline & Adjuvant (Surfactant) & gallon & 22.50 & \\
\hline
\end{tabular}

Source: $\quad$ Ronald P. Muraro, Extension Farm Management Economist, University of Florida, Citrus Research and Education Center (CREC), Lake Alfred, Florida, August 2004. 
Table 10-A.--2004 summary of average fertilizer price estimates

\begin{tabular}{|c|c|c|c|}
\hline Item & Unit & $\begin{array}{c}\text { Average } \\
\text { Price }\end{array}$ & $\begin{array}{c}\text { Your Price } \\
(2004)\end{array}$ \\
\hline \multicolumn{4}{|c|}{ FERTILIZER (FOB Price @ Plant) } \\
\hline & & $\$$ & \\
\hline \multicolumn{4}{|l|}{$\underline{\text { Dry Mix (Bulk) }}$} \\
\hline $17-0-17-3_{\mathrm{Mg}}$ & ton & 196.99 & \\
\hline $17-4-17-2.4_{\mathrm{Mg}}$ & ton & 200.79 & \\
\hline $16-0-16$ & ton & 181.72 & \\
\hline $16-0-16-4_{\mathrm{Mg}}$ & ton & 200.13 & \\
\hline $16-2-16-3_{\mathrm{Mg}}$ & ton & 198.99 & \\
\hline $15-2-15-2.4_{\mathrm{Mg}}$ & ton & 183.26 & \\
\hline $12-2-12-2 \cdot 4_{\mathrm{Mg}}$ & ton & 176.29 & \\
\hline 8-8-8 w/minors* & ton & 169.82 & \\
\hline $8-4-8 \mathrm{w} /$ minors* & ton & 155.77 & \\
\hline $8-2-8 \mathrm{w} /$ minors* & ton & 141.94 & \\
\hline 6-6-6 w/minors* & ton & 150.77 & \\
\hline \multicolumn{4}{|l|}{$\underline{\text { Liquid Mix (Bulk) }}$} \\
\hline $8-2-8$ & ton & 126.89 & \\
\hline $8-4-8$ & ton & 126.55 & \\
\hline $9-3-9$ & ton & 130.31 & \\
\hline $9-4-9$ & ton & 138.77 & \\
\hline $10-0-10$ & ton & 139.81 & \\
\hline $10-2-10$ & ton & 141.62 & \\
\hline $12-0-6$ & ton & 143.28 & \\
\hline $12-3-6$ & ton & 150.88 & \\
\hline
\end{tabular}

*With organic nitrogen, the price averaged $25 \%$ higher. 
Table 10-A.--2004 summary of average fertilizer price estimates (continued)

\begin{tabular}{|c|c|c|c|}
\hline Item & Unit & $\begin{array}{c}\text { Average } \\
\text { Price }\end{array}$ & $\begin{array}{c}\text { Your Price } \\
(2004)\end{array}$ \\
\hline \multicolumn{4}{|l|}{$\underline{\text { Other Fertilizer Materials (Bulk) }}$} \\
\hline Ammonium Nitrate (21\% N Liquid) & ton & 168.83 & \\
\hline Ammonium Nitrate (33.5\% N Dry) & ton & 235.60 & \\
\hline Ammonium Sulfate $(21 \% \mathrm{~N})$ & ton & 147.92 & \\
\hline Calcium Nitrate $(19 \% \mathrm{Ca}, 15.5 \% \mathrm{~N})$ & ton & 223.85 & \\
\hline Dolomite (at mine-- $49 \% \mathrm{CaCO}_{3}, 36 \% \mathrm{MgCO}_{3}$ ) & ton & 15.90 & \\
\hline Muriate of Potash $\left(60 \% \mathrm{~K}_{2} \mathrm{O}\right)$ & ton & 183.78 & \\
\hline Potassium Nitrate $\left(14 \% \mathrm{~N} ; 46 \% \mathrm{~K}_{2} \mathrm{O}\right)$ & ton & 370.75 & \\
\hline Sul-Po-Mag (SPM--21.9\% $\left.\mathrm{K}_{2} \mathrm{O}\right)$ & ton & 183.33 & \\
\hline Super Phosphate $\left(20 \% \mathrm{P}_{2} \mathrm{O}_{5}\right)$ & ton & 204.17 & \\
\hline Triple Superphosphate $\left(48 \% \mathrm{P}_{2} \mathrm{O}_{5}\right)$ & ton & 225.36 & \\
\hline Average Delivery Cost & ton & 12.78 & \\
\hline \multicolumn{4}{|l|}{$\underline{\text { Foliar Macronutrients }}$} \\
\hline Phos Might 0-22-20 & gallon & 24.87 & \\
\hline Nutriphite Magnum 2-40-16 & gallon & 30.00 & \\
\hline MKP (0-52-34) (Mono-Potassium Phosphate) & pound & 0.65 & \\
\hline
\end{tabular}

**SRN, Slow Release Nitrogen

Source: $\quad$ Ronald P. Muraro, Extension Farm Management Economist, University of Florida, Citrus Research and Education Center (CREC), Lake Alfred, Florida, August 2004. 
Table 11-A.--A listing of estimated comparative Central Florida (Ridge) citrus production costs per acre for 2003-2004

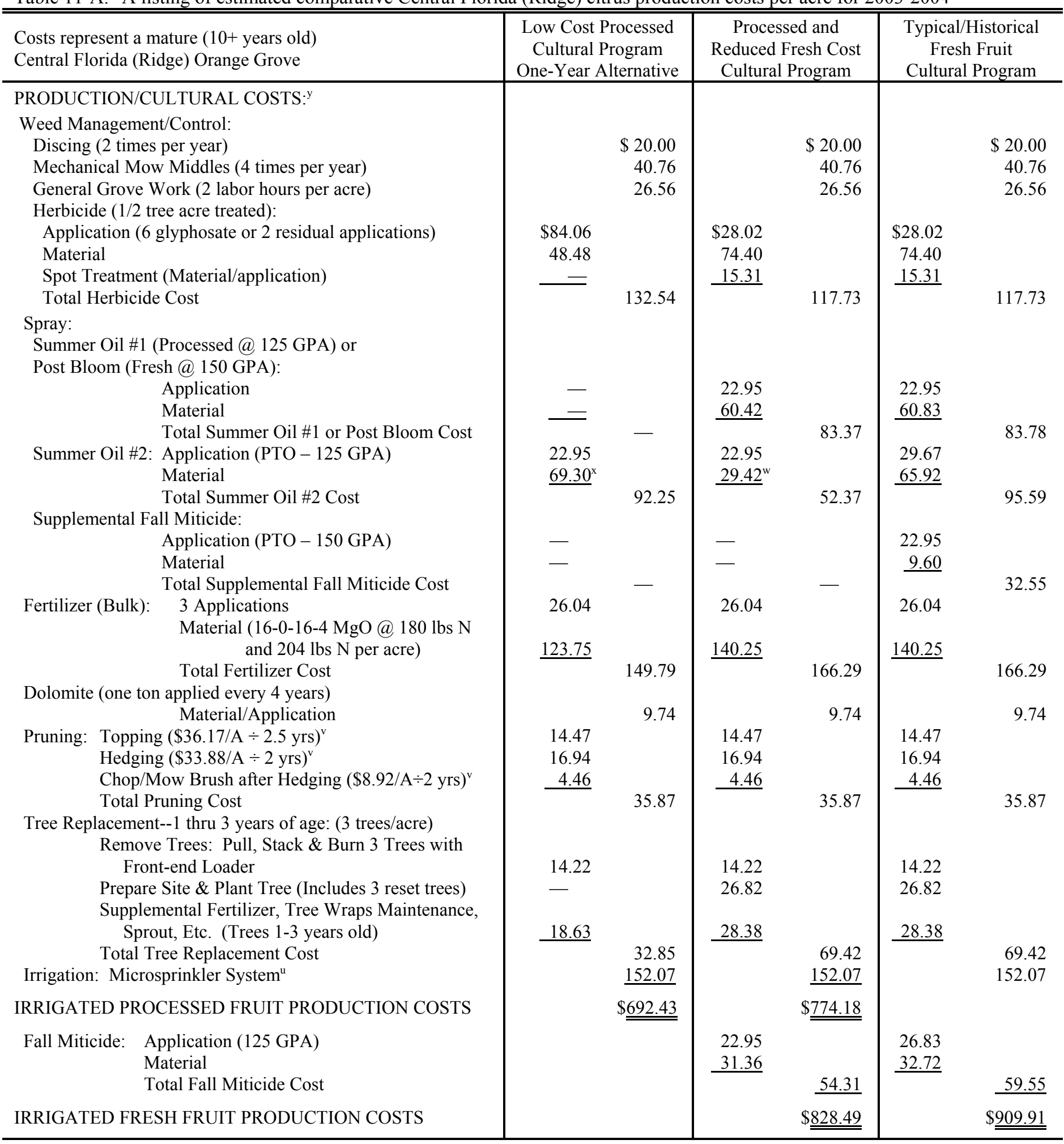

${ }^{2}$ Estimated comparative costs are for example grove situation described in Economic Information Report Series, Budgeting Costs and Returns for Central Florida Citrus Production, and may not represent your particular grove situation in Central Florida.

Source: Ronald P. Muraro, University of Florida-, Citrus Research and Education Center, Lake Alfred, FL, August 2004. 
Table 12-A--Estimated cost of planting and maintaining a reset citrus tree through three years of age, July 2004

\begin{tabular}{|c|c|c|c|c|c|}
\hline & \multicolumn{5}{|c|}{ Number of Resets/Replacement Trees Per Acre } \\
\hline & $1-2$ & $3-5$ & $6-10$ & $11-25$ & $26+$ \\
\hline \multicolumn{6}{|c|}{ - Cost Per Tree } \\
\hline Year \#1: & $\$$ & $\$$ & $\$$ & $\$$ & $\$$ \\
\hline Tree Removal & 5.45 & 4.74 & 3.79 & 3.07 & 2.45 \\
\hline Tree Cost (Container Tree) & 4.50 & 4.50 & 4.35 & 4.25 & 4.25 \\
\hline Site Preparation ${ }^{\mathrm{a}}$ & 5.71 & 4.95 & 4.19 & 3.88 & 3.04 \\
\hline Plant Tree and First Watering & $\underline{2.84}$ & $\underline{2.46}$ & $\underline{2.08}$ & 1.93 & $\underline{1.51}$ \\
\hline Total Planting Cost & $\overline{13.05}$ & $\overline{11.91}$ & $\overline{10.62}$ & $\overline{10.06}$ & $\overline{8.80}$ \\
\hline \multicolumn{6}{|l|}{ Supplemental Fertilization -4 Times } \\
\hline \multicolumn{6}{|l|}{ Supplemental Spraying } \\
\hline (Application \& Materials) $^{\mathrm{b}}$ & 0.48 & 0.41 & 0.38 & 0.35 & 0.32 \\
\hline Spot Herbicide (Application \& Materials) & 0.21 & 0.18 & 0.16 & 0.14 & 0.13 \\
\hline Tree Wrap (Corrugated) & 1.00 & 1.00 & 1.00 & 1.00 & 1.00 \\
\hline Sprouting/Pruning & 0.42 & 0.42 & 0.39 & 0.39 & 0.35 \\
\hline Miscellaneous & 0.17 & 0.16 & 0.15 & 0.14 & 0.14 \\
\hline Supervision \& Overhead & $\underline{0.27}$ & $\underline{0.25}$ & $\underline{0.24}$ & $\underline{0.23}$ & $\underline{0.21}$ \\
\hline Total Tree Care Cost Year \#1 & $\overline{3.92}$ & $\overline{3.62}$ & $\overline{3.42}$ & $\overline{3.25}$ & $\overline{3.07}$ \\
\hline Total Cost Year \#1 & 22.42 & 20.27 & 17.83 & 16.38 & 14.32 \\
\hline \multicolumn{6}{|l|}{ Year \#2: } \\
\hline \multicolumn{6}{|l|}{ Supplemental Fertilization -3 Times } \\
\hline (Application \& Materials) & 1.81 & 1.61 & 1.41 & 1.19 & 1.09 \\
\hline \multicolumn{6}{|l|}{ Supplemental Spraying } \\
\hline (Application \& Materials) $^{\mathrm{b}}$ & 0.55 & 0.49 & 0.43 & 0.36 & 0.33 \\
\hline Spot Herbicide (Application \& Materials) & 0.20 & 0.18 & 0.16 & 0.14 & 0.13 \\
\hline Sprouting/Pruning & 0.50 & 0.50 & 0.42 & 0.42 & 0.38 \\
\hline Miscellaneous & 0.15 & 0.14 & 0.12 & 0.11 & 0.10 \\
\hline Supervision \& Overhead & $\underline{0.24}$ & $\underline{0.20}$ & $\underline{0.19}$ & $\underline{0.17}$ & $\underline{0.15}$ \\
\hline Total Cost Year \#2 & $\overline{3.45}$ & $\overline{3.12}$ & 2.73 & 2.39 & 2.18 \\
\hline \multicolumn{6}{|l|}{$\underline{\text { Year \#3: }}$} \\
\hline \multicolumn{6}{|l|}{ Supplemental Fertilization -3 Times } \\
\hline (Application \& Materials) & 2.48 & 2.21 & 1.90 & 1.62 & 1.37 \\
\hline Miscellaneous & 0.12 & 0.11 & 0.09 & 0.08 & 0.07 \\
\hline Supervision \& Overhead & $\underline{0.20}$ & $\underline{0.17}$ & $\underline{0.15}$ & $\underline{0.13}$ & $\underline{0.11}$ \\
\hline Total Cost Year \#3 ${ }^{b}$ & $\overline{2.80}$ & $\overline{2.49}$ & $\overline{2.14}$ & $\overline{1.83}$ & $\overline{1.55}$ \\
\hline Total Three-Year Cumulative Costs & $\underline{28.67}$ & $\underline{\underline{25.88}}$ & $\underline{\underline{22.70}}$ & $\underline{\underline{20.60}}$ & $\underline{\underline{18.05}}$ \\
\hline
\end{tabular}

${ }^{a}$ Site preparation for bedded citrus grove; cost of root removal, rotovating/leveling tree planting site. Fumigate planting site would cost approximately $\$ 2.50$ per tree.

${ }^{\mathrm{b}}$ Additional spray costs may be incurred if leafminer is a problem.

Source: Ronald P. Muraro, Farm Management Economist, CREC, Lake Alfred, FL, July 2004. 
Table 13-A.--Estimated average picking, roadsiding and hauling charges for Florida citrus, 2003-04

\begin{tabular}{|c|c|c|c|c|c|c|}
\hline & \multicolumn{3}{|c|}{ Fresh Fruit } & \multicolumn{3}{|c|}{ Processed Fruit } \\
\hline & & Range & Average & & Range & Average \\
\hline & & $\$ /$ Box & $\$ /$ Box & & $\$ /$ Box & $\$ /$ Box \\
\hline \multicolumn{7}{|l|}{$\underline{\text { Picking Charges: }}$} \\
\hline Early and Mid-Season Oranges & 0.80 & $-\quad 1.00$ & 0.840 & 0.65 & -0.95 & 0.772 \\
\hline Valencia Oranges & 0.80 & -1.00 & 0.840 & 0.65 & - 0.95 & 0.791 \\
\hline Pink/Red Grapefruit & 0.60 & -0.85 & 0.658 & 0.55 & $-\quad 0.70$ & 0.590 \\
\hline White/Marsh Grapefruit & 0.60 & - $\quad 0.70$ & 0.633 & 0.55 & - $\quad 0.70$ & 0.590 \\
\hline Temples/Tangelos & 0.85 & -1.25 & 0.950 & 0.70 & -1.25 & 0.851 \\
\hline \multirow[t]{4}{*}{ Tangerines } & 1.35 & $-\quad 1.75$ & 1.563 & & - & - \\
\hline & \multicolumn{3}{|c|}{ Fresh Fruit } & \multicolumn{3}{|c|}{ Processed Fruit } \\
\hline & \multicolumn{2}{|c|}{ Range } & Average & \multicolumn{2}{|r|}{ Range } & Average \\
\hline & \multicolumn{2}{|c|}{$\$ /$ Box } & $\$ /$ Box & \multicolumn{2}{|r|}{$\$ /$ Box } & $\$ /$ Box \\
\hline \multicolumn{7}{|l|}{ Roadsiding Charges: } \\
\hline Early and Mid-Season Oranges & 0.70 & -1.06 & 0.860 & 0.65 & $-\quad 1.07$ & 0.801 \\
\hline Valencia Oranges & 0.75 & -1.06 & 0.868 & 0.65 & -1.07 & 0.817 \\
\hline Pink/Red Grapefruit & 0.65 & - $\quad 0.87$ & 0.766 & 0.55 & - 0.66 & 0.620 \\
\hline White/Marsh Grapefruit & 0.65 & -0.85 & 0.743 & 0.55 & - 0.66 & 0.620 \\
\hline Temples/Tangelos & 0.75 & $-\quad 1.11$ & 0.938 & 0.75 & $-\quad 1.07$ & 0.833 \\
\hline \multirow[t]{4}{*}{ Tangerines } & 1.12 & $-\quad 1.21$ & 1.155 & & - & - \\
\hline & \multicolumn{3}{|c|}{ Fresh Fruit } & \multicolumn{3}{|c|}{ Processed Fruit } \\
\hline & \multicolumn{3}{|c|}{ All Varieties } & \multicolumn{3}{|c|}{ All Varieties } \\
\hline & \multicolumn{3}{|c|}{$\$ /$ Box } & \multicolumn{3}{|c|}{$\$ /$ Box } \\
\hline \multicolumn{7}{|l|}{ Hauling Charges: } \\
\hline 0 - 30 miles & \multicolumn{3}{|c|}{0.410} & \multicolumn{3}{|c|}{0.392} \\
\hline $31-50$ miles & \multicolumn{3}{|c|}{0.460} & \multicolumn{3}{|c|}{0.457} \\
\hline $51-80$ miles & \multicolumn{3}{|c|}{0.553} & \multicolumn{3}{|c|}{0.530} \\
\hline $81-100$ miles & \multicolumn{3}{|c|}{0.625} & \multicolumn{3}{|c|}{0.570} \\
\hline $100+$ miles & \multicolumn{3}{|c|}{0.687} & \multicolumn{3}{|c|}{0.625} \\
\hline
\end{tabular}


Table 14-A.--Estimated average packing charges for Florida citrus, 2003-04

\begin{tabular}{|c|c|c|c|c|c|}
\hline & $\begin{array}{l}\text { Domestic } \\
\text { Grapefruit }\end{array}$ & $\begin{array}{c}\text { Export } \\
\text { Grapefruit }\end{array}$ & Oranges & $\begin{array}{l}\text { Temples/ } \\
\text { Tangelos }\end{array}$ & Tangerines \\
\hline \multirow[b]{2}{*}{ Total Packing Charge ${ }^{\mathrm{a}}$} & & ---- & \multirow{2}{*}{$\begin{array}{c}\text { \$/Carton } \\
3.993\end{array}$} & --------- & \multirow[b]{2}{*}{4.795} \\
\hline & 3.636 & 3.741 & & 4.026 & \\
\hline & \multicolumn{3}{|c|}{ - \$Box } & ------ & --- \\
\hline Drenching Charge & 0.158 & 0.158 & 0.173 & 0.173 & 0.173 \\
\hline $\begin{array}{l}\text { Packinghouse Elimination } \\
\text { Charges }\end{array}$ & 0.523 & 0.523 & 0.544 & 0.544 & 0.544 \\
\hline $\begin{array}{l}\text { Hauling Charges for } \\
\text { Eliminations }\end{array}$ & 0.423 & 0.423 & 0.496 & 0.496 & 0.496 \\
\hline
\end{tabular}

${ }^{a}$ Total Packing Charge includes the following items:

1. Materials, including mesh/plastic bags, labels/PLUs, etc.

2. Includes supervisor/foreman labor, grading, palletizing, shipping and general labor. Includes payroll taxes (FICA), workers' compensation, ground insurance, etc.

3. Other direct packing costs include fruit treating; power, lights and water; repairs maintenance; miscellaneous supplies; etc.

4. Indirect packing costs include items such as insurance-fire and casualty; taxes and licenses; depreciation and rent.

5. G\&A costs include office personnel (FICA, w/comp); packinghouse and general manager; office supplies; telephone; etc.

6. Selling Expenses include sales salaries, travel, telephone and telegraph and brokerage fees.

7. Special assessments include items such as advertising taxes; inspection fees; Florida Citrus Packers; CAC.

Note: Packing charges represent a total of nine citrus packinghouses from both the Indian River and Interior Production regions.

Source: $\quad$ Ronald P. Muraro, University of Florida, Citrus Research and Education Center, Lake Alfred, FL, August 2004. 
Table 15-A.--Historic prices ${ }^{\mathrm{a}}$ for selected citrus varieties

\begin{tabular}{|c|c|c|c|c|c|c|c|}
\hline \multirow[b]{3}{*}{ Crop year } & \multicolumn{7}{|c|}{ Variety } \\
\hline & \multirow{2}{*}{$\begin{array}{c}\text { Early }^{\mathrm{b}} \text { and } \\
\text { Mid }^{\mathrm{c}} \text {-season } \\
\text { Oranges } \\
\end{array}$} & \multirow{2}{*}{$\begin{array}{l}\text { Late Season } \\
\text { Oranges }\end{array}$} & \multirow{2}{*}{$\begin{array}{l}\text { Temple } \\
\text { Oranges }\end{array}$} & \multirow{2}{*}{$\begin{array}{c}\text { All } \\
\text { Tangerines }\end{array}$} & \multirow[b]{2}{*}{ Tangelos } & \multicolumn{2}{|c|}{ Seedless Grapefruit $^{\mathrm{e}}$} \\
\hline & & & & & & (white) & (colored) \\
\hline $1961-62$ & $\$ 1.93$ & $\$ 1.81$ & $\$ 2.17$ & $\$ 2.04$ & $\$ 3.36$ & $\$ 0.68$ & $\$ 0.86$ \\
\hline $1962-63$ & 2.17 & 3.50 & 3.09 & 3.02 & 4.66 & 1.29 & 1.81 \\
\hline $1963-64$ & 4.43 & 4.45 & 4.45 & 3.18 & 4.83 & 2.24 & 2.54 \\
\hline $1964-65$ & 2.57 & 2.28 & 2.77 & 2.68 & 4.00 & 1.51 & 1.82 \\
\hline $1965-66$ & 1.44 & 1.79 & 1.80 & 2.14 & 2.85 & 1.39 & 1.64 \\
\hline $1966-67$ & 0.81 & 1.08 & 0.88 & 1.06 & 1.64 & 0.73 & 0.94 \\
\hline $1967-68$ & 1.86 & 2.28 & 2.79 & 4.29 & 3.22 & 2.05 & 2.48 \\
\hline $1968-69$ & 1.56 & 1.83 & 2.22 & 2.55 & 2.47 & 0.98 & 1.15 \\
\hline $1969-70$ & 1.15 & 1.13 & 1.47 & 2.23 & 1.13 & 1.72 & 1.92 \\
\hline $1970-71$ & 1.10 & 1.91 & 1.91 & 1.88 & 1.04 & 1.89 & 2.15 \\
\hline $1971-72$ & 1.98 & 2.11 & 1.95 & 2.97 & 1.69 & 2.27 & 2.69 \\
\hline $1972-73$ & 1.43 & 1.71 & 1.95 & 2.37 & 1.39 & 2.06 & 2.53 \\
\hline $1973-74$ & 1.38 & 1.59 & 1.64 & 2.82 & 1.25 & 1.58 & 2.12 \\
\hline $1974-75$ & 1.46 & 1.82 & 1.68 & 3.05 & 1.45 & 1.55 & 2.59 \\
\hline $1975-76$ & 1.69 & 1.88 & 1.79 & 3.02 & 1.42 & 1.29 & 2.23 \\
\hline $1976-77$ & 1.89 & 2.63 & 2.16 & 3.29 & 1.42 & 1.49 & 2.04 \\
\hline $1977-78$ & 3.90 & 4.40 & 3.92 & 4.79 & 3.29 & 1.47 & 2.09 \\
\hline $1978-79$ & 4.44 & 4.95 & 4.89 & 4.99 & 3.90 & 2.21 & 3.13 \\
\hline $1979-80$ & 3.59 & 3.89 & 2.89 & 4.25 & 2.87 & 3.12 & 3.80 \\
\hline $1980-81$ & 3.67 & 4.63 & 4.21 & 5.45 & 3.92 & 3.46 & 4.22 \\
\hline $1981-82$ & 4.27 & 4.29 & 4.01 & 6.23 & 3.58 & 1.92 & 2.80 \\
\hline $1982-83$ & 4.88 & 5.41 & 3.99 & 7.57 & 4.37 & 1.51 & 3.20 \\
\hline $1983-84$ & 5.09 & 6.72 & 5.34 & 5.93 & 4.28 & 2.08 & 4.05 \\
\hline $1984-85$ & 7.30 & 6.88 & 5.59 & 15.91 & 7.08 & 3.02 & 4.84 \\
\hline $1985-86$ & 3.92 & 3.97 & 3.01 & 12.69 & 4.06 & 3.56 & 4.98 \\
\hline $1986-87$ & 4.56 & 6.02 & 3.60 & 10.92 & 3.72 & 4.45 & 5.80 \\
\hline $1987-88$ & 6.72 & 8.73 & 5.69 & 12.99 & 5.58 & 5.35 & 5.93 \\
\hline $1988-89$ & 6.63 & 8.41 & 5.46 & 12.64 & 6.31 & 4.33 & 4.71 \\
\hline $1989-90$ & 6.01 & 6.53 & 5.64 & 15.28 & 5.10 & 5.21 & 6.30 \\
\hline $1990-91$ & 5.38 & 6.58 & 6.31 & 17.10 & 6.11 & 4.59 & 6.85 \\
\hline $1991-92$ & 5.44 & 6.65 & 6.51 & 18.00 & 7.16 & 6.46 & 6.87 \\
\hline $1992-93$ & 3.23 & 3.88 & 2.99 & 13.75 & 3.31 & 2.22 & 3.11 \\
\hline $1993-94$ & 3.76 & 4.61 & 2.73 & 9.83 & 2.38 & 3.23 & 3.38 \\
\hline $1994-95$ & 3.25 & 4.41 & 3.47 & 11.98 & 2.64 & 2.58 & 1.66 \\
\hline $1995-96$ & 3.62 & 5.57 & 4.44 & 12.59 & 3.63 & 2.14 & 1.77 \\
\hline 1996-97 & 3.18 & 4.07 & 3.22 & 7.99 & 2.19 & 1.12 & 1.91 \\
\hline 1997-98 & 2.81 & 4.88 & 3.07 & 8.49 & 1.66 & 0.93 & 1.50 \\
\hline 1998-99 & 4.35 & 5.58 & 5.12 & 12.07 & 4.53 & 1.95 & 2.65 \\
\hline 1999-00 & 3.19 & 4.33 & 2.55 & 6.67 & 2.52 & 3.87 & 3.36 \\
\hline $2000-01$ & 2.60 & 4.02 & 2.05 & 6.40 & 1.27 & 2.07 & 2.28 \\
\hline 2001-02 & 2.88 & 4.20 & 2.19 & 7.81 & 2.47 & 1.96 & 2.54 \\
\hline $2002-03^{f}$ & 2.81 & 3.95 & 2.35 & 8.53 & 3.23 & 1.62 & 2.49 \\
\hline
\end{tabular}

${ }^{\mathrm{a}}$ On-tree average price per box (1-3/5 bushel box equivalent) for all methods of sale minus pick and haul charges.

${ }^{b}$ Navel and Hamlin $\quad{ }^{c}$ Parson Brown and Pineapple $\quad{ }^{d}$ Valencia $\quad{ }^{e}$ Marsh (white) or pink $\quad{ }^{\text {f Preliminary }}$ Source: Florida Agricultural Statistics Service. 
Table 16-A.--Debt which can be supported per $\$ 1,000.00$ annual payment capacity

\begin{tabular}{|c|c|c|c|c|c|c|c|c|c|c|c|c|c|c|c|}
\hline \multirow{2}{*}{$\begin{array}{l}\text { Loan } \\
\text { Term } \\
\text { (years) } \\
\end{array}$} & \multicolumn{15}{|c|}{ Interest Rate Paid on the Loan } \\
\hline & $8.0 \%$ & $8.5 \%$ & $9.0 \%$ & $9.5 \%$ & $10.0 \%$ & $10.5 \%$ & $11.0 \%$ & $11.5 \%$ & $12.0 \%$ & $12.5 \%$ & $13.0 \%$ & $13.5 \%$ & $14.0 \%$ & $14.5 \%$ & $15.0 \%$ \\
\hline 1 & 926 & 922 & 917 & 913 & 909 & 905 & 901 & 897 & 893 & 889 & 885 & 881 & 877 & 873 & 870 \\
\hline 2 & 1,783 & 1,771 & 1,759 & 1,747 & 1,754 & 1,724 & 1,713 & 1,701 & 1,690 & 1,679 & 1,668 & 1,657 & 1,647 & 1,636 & 1,626 \\
\hline 3 & 2,577 & 2,554 & 2,531 & 2,509 & 2,487 & 2,465 & 2,444 & 2,423 & 2,402 & 2,381 & 2,361 & 2,341 & 2,322 & 2,302 & 2,283 \\
\hline 4 & 3,312 & 3,276 & 3,240 & 3,204 & 3,170 & 3,136 & 3,102 & 3,070 & 3,037 & 3,006 & 2,974 & 2,944 & 2,914 & 2,884 & 2,855 \\
\hline 5 & 3,993 & 3,941 & 3,890 & 3,840 & 3,791 & 3,743 & 3,696 & 3,650 & 3,605 & 3,561 & 3,517 & 3,475 & 3,433 & 3,392 & 3,352 \\
\hline 6 & 4,623 & 4,554 & 4,486 & 4,420 & 4,355 & 4,292 & 4,230 & 4,170 & 4,111 & 4,054 & 3,998 & 3,942 & 3,889 & 3,836 & 3,784 \\
\hline 7 & 5,206 & 5,119 & 5,033 & 4,950 & 4,868 & 4,789 & 4,712 & 4,640 & 4,564 & 4,492 & 4,423 & 4,355 & 4,288 & 4,224 & 4,160 \\
\hline 8 & 5,747 & 5,639 & 5,535 & 5,433 & 5,335 & 5,239 & 5,146 & 5,056 & 4,968 & 4,882 & 4,799 & 4,718 & 4,639 & 4,562 & 4,487 \\
\hline 9 & 6,247 & 6,119 & 5,995 & 5,875 & 5,759 & 5,646 & 5,537 & 5,431 & 5,328 & 5,228 & 5,132 & 5,038 & 4,946 & 4,858 & 4,772 \\
\hline 10 & 6,710 & 6,561 & 6,418 & 6,279 & 6,145 & 6,015 & 5,889 & 5,768 & 5,650 & 5,536 & 5,426 & 5,319 & 5,216 & 5,116 & 5,019 \\
\hline 11 & 7,139 & 6,969 & 6,805 & 6,647 & 6,495 & 6,348 & 6,207 & 6,070 & 5,938 & 5,810 & 5,687 & 5,568 & 5,453 & 5,341 & 5,234 \\
\hline 12 & 7,536 & 7,345 & 7,161 & 6,984 & 6,814 & 6,650 & 6,492 & 6,341 & 6,194 & 6,054 & 5,918 & 5,787 & 5,660 & 5,538 & 5,421 \\
\hline 13 & 7,904 & 7,691 & 7,487 & 7,291 & 7,103 & 6,923 & 6,750 & 6,583 & 6,424 & 6,270 & 6,122 & 5,979 & 5,842 & 5,710 & 5,583 \\
\hline 14 & 8,244 & 8,010 & 7,786 & 7,572 & 7,367 & 7,170 & 6,982 & 6,801 & 6,628 & 6,462 & 6,302 & 6,149 & 6,002 & 5,861 & 5,724 \\
\hline$\underline{15}$ & 8,559 & 8,304 & 8,061 & 7,828 & 7,606 & 7,394 & 7,191 & $\underline{6,997^{\mathrm{a}}}$ & 6,811 & 6,633 & 6,462 & 6,299 & 6,142 & 5,992 & 5,847 \\
\hline$\overline{16}$ & 8,851 & 8,576 & 8,313 & 8,062 & 7,824 & 7,596 & 7,379 & $\overline{7,172}$ & 6,974 & 6,785 & 6,604 & 6,431 & 6,265 & 6,106 & 5,954 \\
\hline 17 & 9,122 & 8,825 & 8,543 & 8,276 & 8,022 & 7,779 & 7,549 & 7,329 & 7,119 & 6,920 & 6,729 & 6,547 & 6,373 & 6,207 & 6,048 \\
\hline 18 & 9,372 & 9,056 & 8,756 & 8,471 & 8,201 & 7,945 & 7,702 & 7,470 & 7,250 & 7,040 & 6,840 & 6,649 & 6,467 & 6,294 & 6,128 \\
\hline 19 & 9,603 & 9,268 & 8,950 & 8,650 & 8,365 & 8,095 & 7,839 & 7,596 & 7,366 & 7,146 & 6,938 & 6,739 & 6,551 & 6,370 & 6,198 \\
\hline$\underline{20}$ & 9,818 & 9,463 & 9,129 & 8,812 & 8,514 & 8,231 & 7,963 & ${\underline{7,710^{\mathrm{a}}}}^{\mathrm{a}}$ & 7,469 & 7,241 & 7,025 & 6,819 & 6,623 & 6,437 & 6,259 \\
\hline 25 & 10,675 & 10,234 & 9,823 & 9,438 & 9,077 & 8,739 & 8,422 & 8,123 & 7,843 & 7,579 & 7,330 & 7,095 & 6,873 & 6,663 & 6,464 \\
\hline 30 & 11,258 & 10,747 & 10,274 & 9,835 & 9,427 & 9,047 & 8,868 & 8,364 & 8,055 & 7,766 & 7,496 & 7,242 & 7,003 & 6,778 & 6,566 \\
\hline 35 & 11,655 & 11,088 & 10,567 & 10,087 & 9,644 & 9,234 & 8,855 & 8,503 & 8,175 & 7,870 & 7,586 & 7,320 & 7,070 & 6,836 & 6,617 \\
\hline 40 & 11,925 & 11,315 & 10,757 & 10,247 & 9,779 & 9,348 & 8,951 & 8,587 & 8,244 & 7,928 & 7,634 & 7,361 & 7,105 & 6,866 & 6,642 \\
\hline
\end{tabular}

${ }^{a}$ Example. Assumes a $\$ 10,000$ after tax income at $11.5 \%$ interest rate and a 15 -year term mortgage, the total debt which can be supported is $\$ 69,970$ $(\$ 6,997 \times 10)$. At $11.5 \%$ interest rate and a 20 -year term mortgage, the total debt which can be supported is $\$ 77,100(\$ 7,710 \times 10)$. 